HEAT TRANSFER- ASIAN RESEARCH

ACCEPTED SEPTEMBER $27^{T H} 2018$

Online ISSN:1523-1496; Publisher - Wiley.

\title{
NUMERICAL STUDY OF HEAT TRANSFER AND VISCOUS FLOW IN A DUAL ROTATING EXTENDABLE DISK SYSTEM WITH A NON-FOURIER HEAT FLUX MODEL
}

\author{
MD. Shamshuddin ${ }^{1 *}$ \\ ${ }^{1 *}$ Department of Mathematics, Vaagdevi College of Engineering, Warangal, Telangana, India. \\ Email: shammaths@gmail.com
}

\author{
S.R. Mishra ${ }^{2}$ \\ ${ }^{2}$ Department of Mathematics, Siksha 'O'Anusandhan Deemed to be University, Bhubaneswar, \\ Odisha, India \\ Email: satyaranjan_mshr@yahoo.co.in
}

\author{
O. Anwar Bég ${ }^{3}$ \\ ${ }^{3}$ Aeronautical and Mechanical Engineering, University of Salford, Manchester, England, UK. \\ Email: gortoab@gmail.com,O.A.Beg@salford.ac.uk
}

\author{
Ali Kadir ${ }^{4}$ \\ ${ }^{4}$ Aeronautical and Mechanical Engineering, School of Computing, Science and Engineering, Newton \\ Building, Salford, M54WT, England, UK. \\ Email: a.Kadir@salford.ac.uk \\ *Corresponding author
}

\begin{abstract}
Nonlinear, steady-state, viscous flow and heat transfer between two stretchable rotating disks spinning at dissimilar velocities is studied with a non-Fourier heat flux model. A non-deformable porous medium is intercalated between the disks and the Darcy model is employed to simulate matrix impedance. The conservation equations are formulated in a cylindrical coordinate system and via the Von Karman transformations are rendered into a system of coupled, nonlinear ordinary differential equations. The emerging boundary value problem is controlled by number of dimensionless dimensionless parameters i.e. Prandtl number, upper disk stretching, lower disk stretching, permeability, non-Fourier thermal relaxation and relative rotation rate parameters. A perturbation solution is developed and the impact of selected parameters on radial and tangential velocity components, temperature, pressure, lower disk radial and tangential skin friction components and surface heat transfer rate are visualized graphically. Validation of solutions with the homotopy analysis method is included. Extensive interpretation of the results is presented which are relevant to to rotating disk bioreactors in chemical engineering.
\end{abstract}

Key words: Rotating disk flow; stretching; heat transfer; viscous hydrodynamics; non-Fourier model; permeability; perturbation method; shooting quadrature; skin friction; radial and tangential flow.

\section{INTRODUCTION}

The viscous flow from a rotating disk (often termed "Von Karman swirling flow") or in the gap between a dual rotating disk system arises in many intriguing applications in the chemical and process engineering industries. These include biological filtration (where porous media feature) [1], spacecraft landing gear lubrication [2], 
rotating cathode electro-chemistry [3], hybrid swirl magnetohydrodynamic propulsion engines [4], photo-catalytic environmental water purification [5], polymer rheometry (for drag reduction studies) [6], hydrogenation processes [7], thermal magnetic materials processing [8], biofuel manufacture [9] and atomization systems [10]. These systems provide a rich arena for hydrodynamic studies. Early investigations of swirling disk flows considered purely viscous hydrodynamics and noteworthy communications in this regard (with a focus on engineering) include Lance and Rogers [11]. Mellor et al. [12] conducted a seminal theoretical (asymptotic), numerical and experimental (hot wire anemometry) investigation of the viscous flow in the region between two coaxial, infinite disks, one rotating and one stationary, identifying that a family of solutions can exist including one-, two- and three-flow cells in the meridional plane. They also demonstrated that the third solution comprises an inwardflowing boundary layer on the stationary disk and an outward-flowing boundary layer on the rotating disk with a core flow intercalated between these two zones which revolves with a constant angular velocity. Furthermore, they obtained the classical single rotating disk solution for infinite Reynolds number as the "free-disk von Kármán solution". More recently Srinivasan and Karra [13] considered the flow between parallel rotating discs with distinct axes as a model of the operation of an orthogonal rheometer. Closed-form solutions for hydromagnetic non-Newtonian flow between off-centred rotating disks were presented by Ersoy [14]. Sha et al. [15] presented asymptotic and computational solutions for the torque and shear stress distributions in dual static-rotating disk flows. Bég et al. [16] computed the velocity and magnetic field distributions in viscous hydromagnetic smart squeeze film flow between rotating disks with the Adomian decomposition method (ADM) for a range of Batchelor magnetic induction numbers.

The above studies did not consider heat or mass transfer. Thermo-solutal transport phenomena are fundamental to numerous chemical engineering processes. The initial work in heat and mass transfer from a rotating disk was presented by Sparrow and Gregg [17] who considered gaseous media and also considered wall suction/blowing effects at the disk surfaces and showed that heat transfer rate is diminished with strong wall injection (blowing). Dorfman [18] presented the first major monograph on boundary layer heat transfer from rotating bodies including disks, cones, spheres and cylinders. Arora and Stokes [19] presented computational solutions for steady axisymmetric flow and dissipative heat transfer of an incompressible Newtonian fluid in the gap between two parallel infinite rotating disks, for different relative rates of rotation and rotational Reynolds numbers up to 125 and Prandtl numbers between 0.7 (air) and 7.5 (water). Hudson [20] derived perturbation solutions for laminar axially symmetric flow and heat transfer between two horizontal disks rotating with a common angular velocity with the upper disk hotter than the lower. He showed that the centrifugal body force generates a tangential flow 
in the thermal layer near the lower disk, that Ekman boundary layers are created at the surface of each disk and that thermal energy is convected by the secondary axial flow. Ming et al. [21] used a multi-shooting method to compute the steady thermal convection flow in non-Newtonian fluid Von Karman swirling flow, observing that the radial velocity is modified with rheological power- law index and that stronger heat conduction to the wall arises with increasing Prandtl number the heat conducts more strongly. Yen and Wang [22] used an orthogonal collocation method to study the combined flow and mass diffusion from a rotating disk against an external forced flow and derived two asymptotic mass-transfer formulae viable for Schmidt number less than 0.1 and greater than unity. Rahman and Postelnicu [23] studied thermophoresis on the forced convective laminar viscous flow from a rotating disk with surface temperature less than the ambient fluid, noting that axial particle deposition velocity increases with thermophoretic coefficient and Schmidt number. Sahoo et al. [24] investigated swirling Von Karman thermal convection flow of a non-Newtonian Reiner-Rivlin fluid. Basu and Cetegen [25] presented an integral analysis of heat transfer in isothermal or iso-flux thin liquid film rotating disk flow surface showing that at weak rotation, surface heat transfer rate decays radially whereas at high rotation, heat transfer peak is displaced radially outwards with greater film Reynolds number. Further studies include Helcig et al. [26] and Jiji and Ganatos [27].

In these studies, the disk boundary has invariably been non-deformable. However, in numerous bio-chemical materials operations (e.g. rotating disk photo-catalytics, bio-membrane manufacture etc), the surface may be stretched or contracted. Important examples of the significance of stretching on hydrodynamics and also thermal/mass diffusion characteristics are provided by Weinstein and Gros [28] (for polymeric extrusion coating systems), Termonia [29] (for heat bonding of non-woven composites for industrial containers) and Khan et al. [30] for high-temperature nano-materials processing. Many theoretical and computational studies of multiphysical stretching boundary transport phenomena have been communicated in recent years. These include Ali $e t$ al. [31] (thermal polymer processing), Bég et al. [32] (magnetic materials processing with cross-diffusion), Abel et al. [33] (time-dependent non-isothermal hydromagnetic extrusion flows), Ahmad et al. [34] (variable thermal conductivity stretching thermal flow), Gupta et al. [35] (unsteady micropolar sheet stretching with wall suction), Yam et al. [36] (rheological flow from stretching wedge geometries), Uddin et al. [37] (high-temperature nanofluid boundary layer slip flows from extending/contracting sheets). Further numerical studies include Sajid et al. [38] (Newtonian viscous flow from curved stretching sheets), Latiff et al. [39] (time-dependent micropolar nanofluid biological slip flows from shrinking or contracting sheets), Hayat et al. [40] viscous thermo-solutal transport from oblique extending cylindrical bodies), Bég et al. [41] gyrotactic nano-bioconvection fully- 
developed flow in stretching/shrinking microchannels, Ali [42] (transpiring heat transfer from a stretched sheet) and Basir et al. [43] (external transient axisymmetric nano-bioconvection slip boundary layers from a stretching pipe). In these studies, different models for stretching were utilized including linear, quadratic, exponential and also power-law and it was shown that stretch rates have a significant influence on skin friction and heat and mass transfer rates at the wall. However, attention was confined to planar (longitudinal) stretching i.e. radial stretching was generally ignored. In disk stretching problems, radial stretching is of primary importance. Several researchers have considered radial stretching effects in both stationary disk flows and Von Karman swirling flows. Bég et al. [44] used a Zhou differential transform algorithm to analyse entropy generation and radial stretching effects on magnetized boundary layer convection from a spinning disk. Hashmi et al. [45] studied the reactive axisymmetric magnetohydrodynamic mixed convection of a viscoelastic nanofluid between two infinite isothermal stretching disks. Fang and Zhang [46] developed closed-form solutions for steady flow between two stretchable infinite disks with accelerated stretching velocity. They noted that a transition in the flow arises from creeping type flow to boundary layer type flow with increasing stretching Reynolds numbers and furthermore that the upper wall stretching parameter markedly influences the velocity distribution. Yin et al. [47] used the Liao homotopy analysis method to compute the impact of disk stretching rate on swirl heat transfer of nanofluids from a rotating disk for a variety of metallic nano-particles. They noted that radial and axial flow and local Nusselt number are enhanced with increasing stretching parameter whereas the tangential flow and thermal boundary layer thickness are reduced. Further studies include Ellahi et al. [48] on magnetic nanofluid flow, heat and mass transfer from an oscillating stretchable rotating disk. Hayat et al. [49] analyzed the dissipative magneto-convective non-Newtonian nanofluid between two rotating stretchable disks.

The classical approach to modelling heat transfer in viscous flows has been the Fourier thermal conduction equation. This approach however reduces the heat conservation formulation to a parabolic energy equation which shows that the medium under observation experiences an initial disturbance. In order to overcome this difficulty, Cattaneo [50] introduced a relaxation time term in Fourier's law of heat conduction which results in the physically realistic finite-speed heat conduction and a hyperbolic model. Following further modification, a modern form of the non-Fourier model which has emerged and has been embraced in engineering sciences is the CattaneoChristov heat flux model. Many excellent studies have utilized this model successfully in a variety of applications including metallurgical solid body heat transfer [51], radiative conduction in solar cells [52], heterogenous materials processing simulations [53] (which identified that a hyperbolic conduction model is appropriate in the bulk material heat transfer), thermal injection moulding polymer processing [54], homogenization of solids [55], 
thermal wave propagation in metallic solids [56]. The Fourier model exhibits infinitesimal heat disturbances which propagate at an infinite speed and is essentially a macroscopic description of solid body heat transfer. However, when there are time delays in heat transfer (in real materials), the non-Fourier (Cattaneo-Christov) model has been shown to accurately capture these effects. Recently this approach has been deployed to simulate thermal conduction effects in thermal convection flows via the inclusion of a thermal relaxation term. Elsayed and Bég [57] used He's variational iteration and Chebyshev's spectral method to compute the non-Fourier effect on biophysical ultrasonic tissue heating. Liu et al. [58] also studied non-Fourier heat conduction in biological materials. Herwig and Beckert [59] considered non-Fourier thermal conduction in complex metallic configurations. Singh et al. [60] presented closed-form solutions for non-Fourier heat transfer in longitudinal fins and heat exchanger systems. Ai and Li [61] used a discontinuous Galerkin finite element method to simulate ultrashort pulsed laser thermal processing of thin films with a non-Fourier heat conduction model, observing significant boundary thermal wave reflection/interactions. Akbar et al. [62] used fourth order Runge-Kutta shooting quadrature to compute the hydromagnetic flow of nanofluids from a stretching surface with the Cattaneo-Christov heat flux model noting that heat transfer rates are substantially altered with non-Fourier thermal relaxation effects. Further studies include Bhatti et al. [63] who simulated the multi-mode non-Fourier heat transfer in electricallyconducting viscoelastic boundary layer flow from an extending sheet noting that increasing thermal relaxation decreases the thermal layer boundary layer thickness. Hayat et al. [64] used the Liao homotopy analysis method to investigate non-Fourier thermal convection in swirling flow between two stretching isothermal disks with a homotopy method.

In the present work, we re-visit and significantly extend the simulations of Hayat et al. [64] to consider the steadystate Newtonian thermal convection flow in the homogenous, high permability, porous medium-filled gap between two stretchable spinning disks rotating at different velocities with the Cattaneo-Christov non-Fourier heat flux model. Viscous-dominated flow is considered and the Darcy model is deployed which is suitable for low Reynolds number regimes. The transformed dimensionless conservation equations are solved subject to appropriate boundary conditions with a perturbation method and evaluated using a Runge-Kutta shooting technique in symbolic software . Further validation with the homotopy analysis method solutions of Hayat $e$ t al. [64] is included. The impact of rotational Reynolds number (based on the spin velocity of the lower disk), Prandtl number, upper disk stretching parameter, lower disk stretching parameter, permeability parameter, non-Fourier thermal relaxation parameter and relative rotation rate parameter on radial and tangential velocity components, temperature, pressure, lower disk radial and tangential skin friction components and surface heat transfer rate are 
visualized graphically and interpretated at length. The solutions provide a solid benchmark for further computational fluid dynamics simulations of relevance to rotating disk bioreactor flows in chemical engineering.

\section{MATHEMATICAL MODEL}

The physical model under consideration is illustrated in Fig.1. We study the axisymmetric viscous thermal convection Von Karman swirling flow in the gap between two stretchable rotating disks in a cylindrical coordinate systems $(r, \theta, z)$. The gap contains an isotropic, homeogenous, high permeability porous medium and Darcy's law is valid. The upper $(z=0)$ and lower disks are separated by a distance $h$. The two disks rotate in the anti-clockwise direction with rotational velocities $\Omega_{1}, \Omega_{1}$. The disks are deformable and are stretched radially with rates $a_{l}$ (lower) and $a_{2}$ (upper). Forced convection takes place between the two disks and the upper disk is maintained at temperature $T_{2}$ and the lower disk at a lesser temperature of $\bar{T}_{1}$. To more accurately simulate heat transfer effects Cattaneo-Christov model for thermal conduction is used. This is a non-Fourier model with additional thermal relxation terms not featured in the classical Fourier model. Edge effects are neglected as are thermal dispersion and stratification. Assuming laminar conditions, the conservation equations for mass (continuity), radial, tangential and axial momentum, and energy conservations with porous medium body forces (Darcian drag) and thermal relaxation, may be shown to take the form [64]:

$$
\begin{aligned}
& \frac{\partial \bar{u}}{\partial r}+\frac{\bar{u}}{r}+\frac{\partial \bar{w}}{\partial z}=0 \\
& \bar{u} \frac{\partial \bar{u}}{\partial r}+\bar{w} \frac{\partial \bar{u}}{\partial z}-\frac{\bar{v}^{2}}{r}=-\frac{1}{\rho} \frac{\partial \bar{p}}{\partial r}+v\left(\frac{\partial^{2} \bar{u}}{\partial r^{2}}+\frac{\partial^{2} \bar{u}}{\partial z^{2}}+\frac{1}{r} \frac{\partial \bar{u}}{\partial r}-\frac{\bar{u}}{r^{2}}\right)-\frac{\mu}{k_{o}} \bar{u} \\
& \bar{u} \frac{\partial \bar{v}}{\partial r}+\bar{w} \frac{\partial \bar{v}}{\partial z}+\frac{\bar{u} \bar{v}}{r}=v\left(\frac{\partial^{2} \bar{v}}{\partial r^{2}}+\frac{\partial^{2} \bar{v}}{\partial z^{2}}+\frac{1}{r} \frac{\partial \bar{v}}{\partial r}-\frac{\bar{v}}{r^{2}}\right)-\frac{\mu}{k_{o}} \bar{v} \\
& \bar{w} \frac{\partial \bar{w}}{\partial r}+\bar{u} \frac{\partial \bar{w}}{\partial z}=-\frac{1}{\rho} \frac{\partial \bar{p}}{\partial z}+v\left(\frac{\partial^{2} \bar{w}}{\partial r^{2}}+\frac{\partial^{2} \bar{w}}{\partial z^{2}}+\frac{1}{r} \frac{\partial \bar{w}}{\partial r}\right)-\frac{\mu_{k}}{k_{o}} \\
& \rho C_{p}\left(\bar{u} \frac{\partial \bar{T}}{\partial r}+\bar{w} \frac{\partial \bar{T}}{\partial z}\right)=-\nabla \cdot q
\end{aligned}
$$

Here $\bar{u}, \bar{v}, \bar{w}$ are the radial, tangential and axial velocity components in the $(r, \theta, z)$ directions respectively, $\bar{T}$ is the temperature, $v$ is the kinematic viscosity, $\bar{p}$ is hydrodynamic pressure of the fluid, $\rho$ is the density of the fluid and $q$ is heat flux. The following initial and boundary conditions are prescribed at disk surfaces [64]: 
$\bar{u}=r a_{1}, \bar{v}=r \Omega_{1}, \bar{w}=0, \bar{T}=\bar{T}_{1}$, at $\quad z=0($ disk 1$)$

$\bar{u}=r a_{2}, \bar{v}=r \Omega_{2}, \bar{w}=0, \bar{T}=\bar{T}_{2}$, at $\quad z=h($ disk 2$\left.)\right\}$

The Cattaneo- Christov [58-64] model defines the heat flux, $q$, according to:

$$
q+\gamma\left[\frac{\partial q}{\partial t}+V . \nabla q-q . \nabla V+(\nabla . V) q\right]=-k \nabla \bar{T}
$$

Here $\gamma$ is the relaxation time for heat flux and $k$ is the temperature-dependent thermal conductivity. Implementing

Eqn. (7) in Eqn (5), the modified heat conservation (thermal) equation assumes the form:

$\bar{u} \frac{\partial \bar{T}}{\partial r}+\bar{w} \frac{\partial \bar{T}}{\partial z}=-\frac{\kappa}{\rho C_{p}}\left(\frac{\partial^{2} \bar{T}}{\partial r^{2}}+\frac{\partial^{2} \bar{T}}{\partial z^{2}}+\frac{1}{r} \frac{\partial \bar{T}}{\partial r}\right)-\gamma\left[\begin{array}{l}\bar{u}^{2} \frac{\partial^{2} \bar{T}}{\partial r^{2}}+\bar{w}^{2} \frac{\partial^{2} \bar{T}}{\partial z^{2}}+2 \bar{u} \bar{w} \frac{\partial^{2} \bar{T}}{\partial z \partial r}+ \\ \left(\bar{u} \frac{\partial \bar{u}}{\partial r}+\bar{w} \frac{\partial \bar{u}}{\partial z}\right) \frac{\partial \bar{T}}{\partial r}+\left(\bar{u} \frac{\partial \bar{w}}{\partial r}+\bar{w} \frac{\partial \bar{w}}{\partial z}\right) \frac{\partial \bar{T}}{\partial z}\end{array}\right]$

Following Fang and Zhang [46], the von Karman similarity transformations may be invoked:

$$
\left.\begin{array}{l}
\bar{u}=r \Omega_{1} f^{\prime}(\eta), \bar{v}=r \Omega_{1} g(\eta), \bar{w}=-2 h \Omega_{1} f(\eta), \\
\theta=\frac{\bar{T}-\bar{T}_{2}}{\bar{T}_{1}-\bar{T}_{2}}, \bar{p}=\rho_{f} \Omega_{1} v_{f}\left(P(\eta)+\frac{1}{2} \frac{r^{2}}{h^{2}} \varepsilon\right), \eta=\frac{z}{h}
\end{array}\right\}
$$

Here the quantities denote respectively dimensionless radial, tangential and axial velocity components, temperature, pressure and axial coordinate. Eqns. (2)-(4), (6) and (8) readily reduce thereby to the following nonlinear, coupled system of ordinary differential equations (the continuity equation is automatically satisfied and also the axial momentum equation is replaced by a pressure equation):

$$
\begin{aligned}
& f^{\prime \prime \prime}+\operatorname{Re}\left(2 f f^{\prime \prime}-f^{\prime 2}+g^{2}-\frac{1}{\beta} f^{\prime}\right)-\varepsilon=0 \\
& \operatorname{Re}\left(2 f^{\prime} g-2 f g^{\prime}+\frac{1}{\beta} g\right)-g^{\prime \prime}=0 \\
& P^{\prime}=\operatorname{Re}\left(\frac{2}{\beta} f-4 f f^{\prime}\right)-2 f^{\prime \prime}=0 \\
& \theta^{\prime \prime}+2 \operatorname{Pr} \operatorname{Re} f \theta^{\prime}-4 \lambda \operatorname{Pr} \operatorname{Re}\left(f^{2} \theta^{\prime \prime}+f f^{\prime} \theta^{\prime}\right)=0
\end{aligned}
$$

The corresponding boundary conditions (6) at the lower and upper disk transform to:

Lower disk: $f(0)=0, f^{\prime}(0)=A_{1}, g(0)=1, \theta(0)=1, P(0)=0$

Upper disk: $f(1)=0, f^{\prime}(1)=A_{2}, g(1)=\tau, \theta(1)=0$ 
Here the non-dimensional parameters are defined as follows: $\operatorname{Re}=h^{2} \Omega_{1} / v_{f}$ denotes rotational Reynolds number (based on the lower disk spin velocity), $\operatorname{Pr}=\left(\rho C_{p}\right)_{f} v_{f} / k_{f}$ is Prandtl number, $A_{1}=a_{1} / \Omega_{1}, A_{2}=a_{2} / \Omega_{2}$ are scaled radial stretching parameters, $\beta=k_{O} \Omega_{1} / v$ is permeability parameter (it is incorrectly termed the "porosity parameter" in Hayat et al. [64]), $\lambda=\gamma \Omega_{1}$ is thermal relaxation (nonFourier) parameter, $\tau=\Omega_{1} / \Omega_{2}$ is relative rotation parameter and $\varepsilon$ is the pressure parameter. Following Fang and Zhang [46] Eqn. (10) may be further simplified by differentiation w.r.t transformed axial coordinate, $\eta$, leading to:

$$
f^{1 v}+\operatorname{Re}\left(2 f f^{\prime \prime \prime}+2 g g^{\prime}-\frac{1}{\beta} f^{\prime \prime}\right)=0
$$

The pressure parameter $\varepsilon$ may be obtained via Eqn. (10) and conditions (14) as follows [46, 64]:

$$
\varepsilon=f^{\prime \prime \prime}(0)-\operatorname{Re}\left(\left(f^{\prime}(0)\right)^{2}-(g(0))^{2}+\frac{1}{\beta} f^{\prime}(0)\right)=0
$$

The pressure function is easily thereafter derived via integration of Eqn. (12) as follows:

$$
P=2\left[\operatorname{Re}\left(f^{2}+\frac{1}{\beta} \int_{0}^{\eta} f d \eta\right)-f^{\prime}+f^{\prime}(0)\right]=0
$$

Several important engineering design quantities may also be defined. The total shear stress at each disk surface is given by:

$$
\tau_{w}=\sqrt{\tau_{z r}^{2}+\tau_{z \theta}^{2}}
$$

where for the lower disk $(\eta=0)$, radial shear stress $\tau_{z r}=\mu\left(\frac{\partial \bar{u}}{\partial z}\right)_{z=0}=\frac{\mu r \Omega_{1}}{h} f^{\prime \prime}(0)$ and tangential shear stress $\tau_{z \theta}=\mu\left(\frac{\partial \bar{v}}{\partial z}\right)_{z=0}=\frac{\mu r \Omega_{1}}{h} g^{\prime}(0)$. Introducing the appropriate dimensionless variables, we arrive at the following expressions for the non-dimensional total skin friction coefficients:

At lower disk: $\left.C_{f 1}=\frac{\left(\tau_{w}\right)_{z=0}}{\rho\left(r \Omega_{1}\right)^{2}}=\frac{1}{\operatorname{Re}_{r}} \sqrt{\left[\left(f^{\prime \prime}(0)\right)^{2}+\left(g^{\prime}(0)\right)^{2}\right.}\right]$

At upper disk: $\left.C_{f 2}=\frac{\left(\tau_{w}\right)_{z=h}}{\rho\left(r \Omega_{2}\right)^{2}}=\frac{1}{\operatorname{Re}_{r}} \sqrt{\left[\left(f^{\prime \prime}(1)\right)^{2}+\left(g^{\prime}(1)\right)^{2}\right.}\right]$

Here $\operatorname{Re}_{r}=r \Omega_{1} h / v$ is a local rotational Reynolds number. 


\section{PERTUBATION SOLUTION}

The non-dimensional ordinary differential Eqns. (10)- (13) along with boundary conditions (14) are initially perturbed into a set of differential equations using the rotational Reynolds number, $R e$, as the perturbation parameter $(\operatorname{Re}<<1)$, following Van Dyke [65]. Defining the expansions for each transformed variable, $f, g, p$ and $\theta$, we have:

$$
\left.\begin{array}{l}
f=f_{0}+\operatorname{Re} f_{1}+\operatorname{Re}^{2} f_{2}+o\left(\operatorname{Re}^{3}\right) \\
g=g_{0}+\operatorname{Re} g_{1}+\operatorname{Re}^{2} g_{2}+o\left(\operatorname{Re}^{3}\right) \\
p=p_{0}+\operatorname{Re} p_{1}+\operatorname{Re}^{2} p_{2}+o\left(\operatorname{Re}^{3}\right) \\
\theta=\theta_{0}+\operatorname{Re} \theta_{1}+\operatorname{Re}^{2} \theta_{2}+o\left(\operatorname{Re}^{3}\right)
\end{array}\right\}
$$

Inserting Eqn. (21) into Eqns. (10) - (13) and the disk surface boundary conditions (14) and equating the coefficient of zeroth, first and second order for $R e$, yields the following differential equations and corresponding boundary conditions:

\section{Zeroth order}

$$
\begin{aligned}
& f_{0}^{\prime \prime \prime}-\varepsilon=0 \\
& g_{0}{ }^{\prime \prime}=0 \\
& p_{0}^{\prime}=-2 f_{0}^{\prime \prime} \\
& \frac{1}{\operatorname{Pr}} \theta_{0}^{\prime \prime}=0 \\
& f_{0}(\eta)=0, f_{0}^{\prime}(\eta)=A_{1}, g_{0}(\eta)=1, p_{0}(\eta)=0, \theta_{0}(\eta)=1, \text { at } \eta=0 \\
& \left.f_{0}(\eta)=0, f_{0}^{\prime}(\eta)=A_{2}, g_{0}(\eta)=\tau, \theta_{0}(\eta)=0 \quad \text { at } \eta=1\right\}
\end{aligned}
$$

\section{First order}

$$
\left.\begin{array}{l}
f_{1}^{\prime \prime \prime}+2 f_{0} f_{0}^{\prime \prime}-f_{0}^{\prime 2}+g_{0}{ }^{2}-\frac{1}{\beta} f_{0}^{\prime}=0 \\
g_{1}^{\prime \prime}-2 f_{0}^{\prime} g_{0}+2 f_{0} g_{0}^{\prime}-\frac{1}{\beta} g_{0}=0 \\
p_{1}^{\prime}=\frac{2}{\beta} f_{0}-4 f_{0} f_{0}^{\prime}-2 f_{1}^{\prime \prime} \\
\frac{1}{\operatorname{Pr}} \theta_{1}^{\prime \prime}+2 f_{0} \theta_{0}^{\prime}-4 \lambda\left(f_{0} \theta_{0}^{\prime \prime}+f_{0} f_{0}^{\prime} \theta_{0}^{\prime}\right)=0
\end{array}\right\}
$$




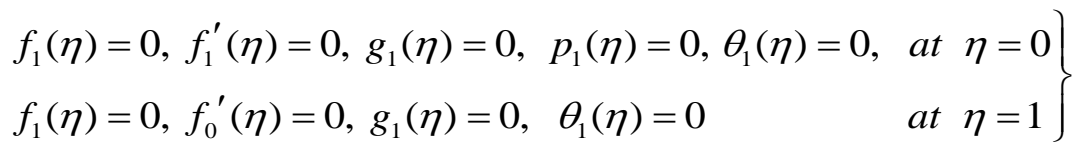

\section{Second order}

$$
\begin{aligned}
& f_{2}^{\prime \prime \prime}+2\left(f_{0} f_{1}^{\prime \prime}+f_{1} f_{0}^{\prime \prime}\right)-2 f_{0}^{\prime} f_{1}^{\prime}+2 g_{0} g_{1}-\frac{1}{\beta} f_{1}^{\prime}=0 \\
& g_{2}^{\prime \prime}-2\left(f_{0}^{\prime} g_{1}+f_{1}^{\prime} g_{0}\right)+2\left(f_{0} g_{1}^{\prime}+f_{1} g_{0}{ }^{\prime}\right)-\frac{1}{\beta} g_{1}=0 \\
& p_{2}^{\prime}=\frac{2}{\beta} f_{1}-4\left(f_{0} f_{1}^{\prime}+f_{1} f_{0}^{\prime}\right)-2 f_{2}^{\prime \prime} \\
& \frac{1}{\operatorname{Pr}} \theta_{2}^{\prime \prime}+2\left(f_{0} \theta_{1}^{\prime}+f_{1} \theta_{0}^{\prime}\right)-4 \lambda\left(f_{0} \theta_{1}^{\prime \prime}+f_{1} \theta_{0}^{\prime \prime}+f_{0} f_{0}^{\prime} \theta_{1}^{\prime}+f_{0} f_{1}^{\prime} \theta_{0}^{\prime}+f_{1} f_{0}^{\prime} \theta_{0}^{\prime}\right)=0 \\
& f_{2}(\eta)=0, f_{2}^{\prime}(\eta)=0, g_{2}(\eta)=0, p_{2}(\eta)=0, \theta_{2}(\eta)=0, \text { at } \eta=0 \\
& \left.f_{2}(\eta)=0, f_{2}^{\prime}(\eta)=0, g_{2}(\eta)=0, \theta_{2}(\eta)=0 \quad \text { at } \eta=1\right\}
\end{aligned}
$$

The set of perturbation Eqns. (22)- (24) and (26) with the boundary conditions (23), (25) and (27) are solved analytically as follows:

\section{Zeroth order solution}

$$
\left.\begin{array}{l}
f_{0}(\eta)=A_{3} \eta^{3}+A_{4} \eta^{2}+A_{1} \eta \\
g_{0}(\eta)=(\tau-1) \eta+1 \\
p_{0}(\eta)=-6 A_{3} \eta^{2}-4 A_{4} \eta \\
\theta_{0}(\eta)=1-\eta
\end{array}\right\}
$$

\section{First order solution}

$$
\left.\begin{array}{l}
f_{1}(\eta)=\frac{9}{210} A_{3}{ }^{2} \eta^{7}+\frac{1}{120} A_{5} \eta^{6}+\frac{1}{60} A_{6} \eta^{5}+\frac{1}{24} A_{7} \eta^{4}+\frac{1}{6} A_{8} \eta^{3}+\frac{1}{2} A_{9} \eta^{2} \\
g_{1}(\eta)=\frac{1}{20} A_{10} \eta^{5}+\frac{1}{12} A_{11} \eta^{4}+\frac{1}{6} A_{12} \eta^{3}+\frac{1}{2} A_{13} \eta^{2}+A_{14} \eta \\
p_{0}(\eta)=-2 A_{3}{ }^{2} \eta^{6}+\frac{1}{5} A_{15} \eta^{5}+\frac{1}{4} A_{16} \eta^{4}+\frac{1}{3} A_{17} \eta^{3}+\frac{1}{2} A_{18} \eta^{2}-2 A_{8} \eta \\
\theta_{0}(\eta)=-\frac{2}{7} \lambda \operatorname{Pr} A_{3}{ }^{2} \eta^{7}+\frac{1}{30} A_{19} \eta^{6}+\frac{1}{20} A_{20} \eta^{5}+\frac{1}{12} A_{21} \eta^{4}+\frac{1}{6} A_{22} \eta^{3}+A_{23} \eta
\end{array}\right\}
$$

\section{Second order solution}




$$
\left.\begin{array}{rl}
f_{2}(\eta) & =A_{24} \eta^{11}+A_{25} \eta^{10}+A_{26} \eta^{9}+A_{27} \eta^{8}+A_{28} \eta^{7}+A_{29} \eta^{6}+A_{30} \eta^{5}+A_{31} \eta^{4}+A_{32} \eta^{3}+A_{33} \eta^{2} \\
g_{2}(\eta) & =A_{34} \eta^{9}+A_{35} \eta^{8}+A_{36} \eta^{7}+A_{37} \eta^{6}+A_{38} \eta^{5}+A_{39} \eta^{4}+A_{40} \eta^{3}+A_{41} \eta \\
p_{2}(\eta) & =A_{42} \eta^{10}+A_{43} \eta^{9}+A_{44} \eta^{8}+A_{45} \eta^{7}+A_{46} \eta^{6}+A_{47} \eta^{5}+A_{48} \eta^{4}+A_{49} \eta^{3}-6 A_{32} \eta^{2}-4 A_{33} \eta \\
\theta_{0}(\eta) & =\frac{1}{156} A_{50} \eta^{13}+\frac{1}{132} A_{51} \eta^{12}+\frac{1}{110} A_{52} \eta^{11}+\frac{1}{90} A_{53} \eta^{10}+\frac{1}{72} A_{54} \eta^{9}+ \\
\frac{1}{56} A_{55} \eta^{8}+\frac{1}{42} A_{56} \eta^{7}+\frac{1}{30} A_{57} \eta^{6}+\frac{1}{40} A_{58} \eta^{5}+\frac{1}{12} A_{59} \eta^{4}+\frac{1}{6} A_{60} \eta^{3}+A_{61} \eta
\end{array}\right\}
$$

Here the following definitions apply:

$$
\begin{aligned}
& A_{3}=\varepsilon / 6, A_{4}=A_{1}-\varepsilon / 6, A_{5}=12 A_{3} A_{4}-12 A_{3}{ }^{2}, A_{6}=4 A_{4}+6 A_{1} A_{3}-12 A_{3} A_{4}-(\tau-1)^{2}+3 / \beta A_{3}, \\
& A_{7}=4 A_{1} A_{4}-12 A_{1} A_{3}-2(\tau-1)+2 / \beta A_{4}, A_{8}=A_{1}^{2}+A_{1} / \beta, A_{9}=-\left(\frac{9}{105} A_{3}^{2}+\frac{A_{5}}{60}+\frac{A_{6}}{30}+\frac{A_{7}}{12}+\frac{A_{8}}{3}\right) \text {, } \\
& A_{10}=4 A_{3}(\tau-1), A_{11}=2 A_{4}(\tau-1)+6 A_{3}, A_{12}=\frac{A_{1}}{\beta}(\tau-1), A_{13}=2 A_{1}+\frac{1}{\beta}, A_{14}=-\left(\frac{A_{10}}{20}+\frac{A_{11}}{12}+\frac{A_{12}}{6}+\frac{A_{13}}{2}\right) \text {, } \\
& A_{15}=-20 A_{3} A_{4}-18 A_{3}, A_{16}=-16 A_{1} A_{3}-8 A_{4}^{2}-2 A_{5}+\frac{2}{\beta} A_{3}, A_{17}=-12 A_{1} A_{4}-2 A_{6}+\frac{2}{\beta} A_{4}, A_{18}=-4 A_{4}^{2}-2 A_{7}+\frac{2}{\beta} A_{1} \text {, } \\
& A_{19}=-8 \lambda \operatorname{Pr}\left(A_{3} A_{4}-A_{4}^{2}\right), A_{20}=\operatorname{Pr} A_{3}\left(2-4 \lambda A_{1}-8 \lambda A_{4}\right), A_{21}=\operatorname{Pr} A_{4}\left(2-12 \lambda A_{1}\right), A_{22}=\operatorname{Pr} A_{1}\left(2-4 \lambda A_{1}\right) \text {, } \\
& A_{23}=-\frac{2}{7} \lambda \operatorname{Pr} A_{3}^{2}+\frac{A_{19}}{30}+\frac{A_{20}}{20}+\frac{A_{21}}{12}+\frac{A_{22}}{6}, A_{24}=\frac{27}{35} A_{3}^{2}, A_{25}=-\frac{3}{10} A_{3} A_{5}-\frac{18}{7} A_{3}^{2} A_{4} \text {, } \\
& A_{26}=\frac{2 A_{3} A_{6}}{15}-\frac{A_{4} A_{5}}{3}-\frac{44 A_{3}^{2} A_{1}}{15}-\frac{(\tau-1) A_{10}}{10}+\frac{9 A_{3}^{2}}{30 \beta}, A_{27}=-\frac{A_{3} A_{7}}{2}+\frac{A_{4} A_{6}}{15}+\frac{A_{1} A_{5}}{10}-\frac{A_{10}}{10}-\frac{(\tau-1) A_{11}}{6}+\frac{A_{5}}{20 \beta}, \\
& A_{28}=-\frac{A_{4} A_{7}}{2}-A_{3} A_{8}-\frac{A_{1} A_{6}}{2}-\frac{44 A_{3}^{2} A_{1}}{15}-\frac{(\tau-1) A_{12}}{3}-\frac{A_{11}}{6}+\frac{A_{16}}{12 \beta}, A_{29}=-\frac{2 A_{1} A_{7}}{3}-\frac{2 A_{3} A_{8}}{3}+A_{3} A_{9}+\frac{A_{12}}{3}-(\tau-1) A_{13}+\frac{A_{7}}{6 \beta}, \\
& A_{30}=-A_{1} A_{8}-2 A_{4} A_{9}-3 A_{3} A_{9}-\frac{A_{13}}{2}-2(\tau-1) A_{14}+\frac{A_{8}}{2 \beta}, A_{31}=-2 A_{1} A_{9}-2 A_{14}, A_{32}=A_{1} A_{9}+\frac{A_{9}}{2 \beta} \text {, } \\
& A_{33}=-\left(\frac{A_{24}}{990}+\frac{A_{25}}{720}+\frac{A_{26}}{504}+\frac{A_{27}}{336}+\frac{A_{28}}{210}+\frac{A_{29}}{120}+\frac{A_{30}}{60}+\frac{A_{31}}{24}+\frac{A_{32}}{6}\right), A_{34}=-\frac{A_{3} A_{10}}{5}+\frac{18(\tau-1) A_{11}}{35}, \\
& A_{35}=-\frac{A_{3} A_{11}}{6}-\frac{3 A_{4} A_{10}}{10}+\frac{9 A_{3}^{2}}{15}-\frac{(\tau-1) A_{5}}{12}, A_{36}=-\frac{A_{4} A_{11}}{3}-\frac{2 A_{1} A_{10}}{5}+\frac{2(\tau-1) A_{6}}{15}+\frac{A_{5}}{10}+\frac{A_{10}}{20 \beta}, \\
& A_{37}=A_{3} A_{13}-\frac{A_{4} A_{12}}{3}-\frac{A_{1} A_{11}}{2}+\frac{(\tau-1) A_{7}}{4}+\frac{A_{6}}{6}+\frac{A_{11}}{12 \beta}, A_{38}=A_{3} A_{14}-\frac{2 A_{1} A_{12}}{3}-\frac{2(\tau-1) A_{8}}{3}+\frac{A_{7}}{3}+\frac{A_{12}}{6 \beta},
\end{aligned}
$$




$$
\begin{aligned}
& A_{39}=2 A_{4} A_{14}-A_{1} A_{13}+(\tau-1) A_{9}+A_{8}+\frac{A_{13}}{2 \beta}, A_{40}=2 A_{9}+\frac{A_{14}}{\beta}, A_{41}=-\left(\frac{A_{34}}{72}+\frac{A_{35}}{56}+\frac{A_{36}}{42}+\frac{A_{37}}{30}+\frac{A_{38}}{20}+\frac{A_{39}}{12}+\frac{A_{40}}{6}\right) \text {, } \\
& A_{42}=-220 A_{24}-\frac{4 A_{3}^{3}}{105}, A_{43}=-180 A_{25}-\frac{A_{3} A_{5}}{5}-\frac{158 A_{4} A_{3}^{2}}{105}-\frac{A_{5}}{10}, A_{44}=-\frac{8 A_{3} A_{6}}{15}-\frac{4 A_{4} A_{5}}{15}-\frac{144 A_{3}{ }^{2} A_{1}}{105}-144 A_{26}+\frac{A_{5}}{60 \beta}, \\
& A_{45}=-4 A_{3} A_{8}-A_{4} A_{7}-\frac{2 A_{1} A_{6}}{5}-84 A_{28}+\frac{A_{6}}{30 \beta}, A_{46}=-10 A_{3} A_{9}-\frac{10 A_{4} A_{8}}{3}-\frac{5 A_{1} A_{7}}{6}-60 A_{29}+\frac{A_{7}}{12 \beta}, \\
& A_{47}=-8 A_{4} A_{9}-\frac{8 A_{1} A_{8}}{3}-40 A_{30}+\frac{A_{8}}{3 \beta}, A_{48}=-6 A_{1} A_{9}-24 A_{31}+\frac{A_{9}}{\beta}, A_{49}=-\left(\frac{A_{42}}{10}+\frac{A_{43}}{9}+\frac{A_{44}}{8}+\frac{A_{45}}{7}+\frac{A_{46}}{6}+\frac{A_{47}}{5}+\frac{A_{48}}{4}+\frac{A_{49}}{3}\right), \\
& A_{50}=-\frac{24}{13} \lambda^{2} \operatorname{Pr}^{2} A_{3}{ }^{4}, A_{51}=\frac{12}{5} \lambda A_{3}{ }^{2} \operatorname{Pr} A_{19}-24 \lambda^{2} A_{3}{ }^{3} \operatorname{Pr}^{2} A_{4}, A_{52}=\lambda \operatorname{Pr}\left(-\frac{57}{21} A_{3}{ }^{3}-8 \lambda A_{3}{ }^{3} \operatorname{Pr} A_{1}+\frac{8}{5} A_{3} A_{4} A_{19}+3 A_{3}{ }^{2} A_{20}\right), \\
& A_{53}=\lambda \operatorname{Pr}\left(\begin{array}{l}
-\frac{2}{5 \lambda} A_{3} A_{19}+4 \operatorname{Pr}-48 \lambda A_{3}^{3} \operatorname{Pr}-8 \lambda \operatorname{Pr} A_{3}^{3} A_{4} A_{1}+\frac{8}{5} A_{19} A_{4}{ }^{2}+\frac{12}{5} A_{3} A_{1} A_{19}+3 A_{3} A_{4} A_{20} \\
-\frac{1}{5} A_{3} A_{5}-\frac{8}{15} A_{3}{ }^{2} A_{4}-\frac{36}{105} A_{3}{ }^{2} A_{4}-\frac{1}{10} A_{3} A_{5}
\end{array}\right), \\
& A_{54}=\lambda \operatorname{Pr}\left(\begin{array}{l}
-\frac{1}{2 \lambda} A_{3} A_{20}-\frac{2}{5 \lambda} A_{4} A_{19}+4 \operatorname{Pr} A_{3}^{2} A_{1}+\frac{8}{5 \lambda} A_{3}^{2}+4 A_{3} A_{19}-48 \lambda \operatorname{Pr} A_{4} A_{3}^{2}-8 \lambda \operatorname{Pr} A_{1}^{2} A_{3}^{2}+\frac{8}{5} A_{4} A_{1} A_{19} \\
+\frac{4}{5} A_{4} A_{1} A_{19}+A_{3} A_{1} A_{20}+\frac{20}{3} A_{3} A_{4} A_{21}+6 A_{3}^{2} A_{22}-\frac{8}{15} A_{3} A_{6}-\frac{4}{15} A_{4} A_{5}-\frac{144}{105} A_{3}^{2} A_{1}
\end{array}\right), \\
& A_{55}=\lambda \operatorname{Pr}\left(\begin{array}{l}
-\frac{2}{3 \lambda} A_{3} A_{21}-\frac{1}{2 \lambda} A_{4} A_{20}-\frac{2}{5 \lambda} A_{1} A_{19}+4 \operatorname{Pr} A_{3} A_{20}+\frac{1}{60 \lambda} A_{5}+4 A_{4} A_{19}-48 \lambda \operatorname{Pr} A_{1} A_{3}^{2}-\frac{4}{3} A_{1} A_{3} A_{21} \\
+\frac{14}{3} A_{4} A_{3} A_{22}+4 A_{4} A_{1} A_{20}+\frac{8}{3} A_{4}^{2} A_{21}-8 \lambda \operatorname{Pr} A_{3}^{2} A_{1}^{2}+2 A_{1} A_{4} A_{20}+4 A_{1} A_{3} A_{21}-\frac{2}{3} A_{3} A_{7}-\frac{1}{5} A_{1} A_{5} \\
-\frac{1}{30} A_{1} A_{5}-\frac{2}{15} A_{4} A_{6}-\frac{1}{2} A_{3} A_{7}
\end{array}\right), \\
& A_{56}=\lambda \operatorname{Pr}\left(\begin{array}{l}
-\frac{1}{\lambda} A_{3} A_{22}-\frac{2}{3 \lambda} A_{4} A_{21}-\frac{1}{2 \lambda} A_{1} A_{20}+4 A_{3} A_{21}+\frac{1}{30 \lambda} A_{6}+4 A_{4} A_{20}+4 A_{1} A_{19}+12 A_{1} A_{3}^{2}+2 A_{1} A_{3} A_{22} \\
+4 A_{4}^{2} A_{22}+\frac{4}{3} A_{4} A_{1} A_{21}+6 A_{1} A_{3} A_{22}+\frac{8}{3} A_{4}{ }^{2} A_{1}+A_{1}^{2} A_{20}-4 A_{3} A_{8}-A_{4} A_{7}+\frac{4}{15} A_{1} A_{6}
\end{array}\right), \\
& A_{57}=\lambda \operatorname{Pr}\left(\begin{array}{l}
-\frac{1}{\lambda} A_{4} A_{22}-\frac{2}{3 \lambda} A_{1} A_{21}+\frac{1}{12 \lambda} A_{7}+4 A_{3} A_{22}+4 A_{4} A_{21}+4 A_{1} A_{20}+20 A_{4} A_{3} A_{23}+4 A_{4} A_{1} A_{22} \\
+\frac{4}{5} A_{1}^{2} A_{21}-10 A_{3} A_{9}-\frac{10}{3} A_{4} A_{8}-\frac{5}{6} A_{7} A_{1}
\end{array}\right), \\
& A_{58}=\lambda \operatorname{Pr}\left(-\frac{2}{\lambda} A_{3} A_{23}-\frac{1}{\lambda} A_{1} A_{22}+\frac{1}{12 \lambda} A_{7}+4 A_{3} A_{23}+4 A_{1} A_{22}-8 A_{4} A_{9}-\frac{8}{3} A_{1} A_{8}\right) \text {, } \\
& A_{59}=\lambda \operatorname{Pr}\left(-\frac{2}{\lambda} A_{4} A_{23}+\frac{1}{\lambda} A_{9}+4 A_{1} A_{22}+6 A_{1} A_{4} A_{23}-6 A_{1} A_{9}\right), A_{60}=-2 A_{1} A_{23} \\
& A_{61}=-\left(\frac{A_{50}}{156}+\frac{A_{51}}{132}+\frac{A_{52}}{110}+\frac{A_{53}}{90}+\frac{A_{54}}{72}+\frac{A_{55}}{56}+\frac{A_{56}}{42}+\frac{A_{57}}{30}+\frac{A_{58}}{20}+\frac{A_{59}}{12}+\frac{A_{60}}{6}\right) \text {. }
\end{aligned}
$$


To validate the present perturbation solutions, we compare with the homotopy solutions of Hayat et al. [64]. For selected values of all the key parameters, we have computed the tangential skin friction component and the wall heat transfer rate (Nusselt number function) in Table 1. Very good correlation is achieved demonstrating high confidence in the present perturbation method.

\section{RESULTS AND DISCUSSION}

Comprehensive solutions have been plotted for all the key fluid and thermal characteristics in Figs. 2-13 and

Table 2. Default values have been prescribed as follows for the seven dictating parameters: $\varepsilon=0.01$ (weak pressure effect), $\beta=0.9$ (high permeability corresponding to industrial metal foams, highly fibrous filtration media etc), $\operatorname{Pr}=0.7$ (air), $A_{l}=0$ (lower disk not stretched), $\tau=0.8$ (upper disk rotates faster than lower disk), $\lambda=0.2$ (low thermal relaxation) and $R e=0.01$ (low rotational Reynolds number).

It is important to note that three rotation scenarios are possible, as simulated via the relative rotation parameter, $\tau$. These are incorrectly defined in Hayat et al. [64]. They are dis-ambiguated here. For the case $\tau>0$, both disks corotate i.e. they rotate in the same direction. When $\tau=0$, the upper disk is stationary $\left(\Omega_{2} \rightarrow 0\right)$ and only the lower disk rotates. When $\tau<0$ both disks rotate in opposite directions to each other i.e. counter-rotate. The most relevant of these scenarios to biochemical disk reactors is the first scenario i.e. co-rotation and is therefore the only one considered here. Also, we note that the upper disk radial stretching rate is fixed at $A_{2}=0.4$ since the lower disk is of primary interest here.

\section{Radial flow characteristics}

Figs 2-5 illustrate the response in radial stream function and radial velocity function (i.e. gradient of radial stream function) to modifications in various parameters. Increasing rotational Reynolds number (fig. 2) is observed to significantly elevate the stream function across the gap. However, there is an increase in skewness of profiles towards the upper disk. The increase in lower disk rotational velocity (to which $R e$ is proportional for constant gap width, $h$, and dynamic viscosity, $v_{f}$ ) generates strong inertia in the lower half space. This leads to an accentuation in the radial velocity magnitudes (fig. 3 ) in this zone and a corresponding deceleration in radial flow in the upper half space, in accordance with conservation of momentum. This is characteristic of swirl flows and has been identified in many other studies including Arora and Stokes [19] and Hudson [20]. The negative radial velocities in the upper half space of the gap in fig. 2 imply that the flow is reversed here i.e. backflow is induced 
and this effect is naturally maximized at the upper disk, as noted also by Lance and Rogers [11]. The trends also confirm the findings of Mellor et al. [12] namely that there is an inner core flow which is rotating with constant angular velocity irrespective of the rotational Reynolds number. The lower disk is surrounded by an outwardflowing boundary layer whereas the upper disk is associated with an inward-flowing boundary layer structure. The fluid structure is therefore similar to an axial fan configuration, although it is asymmetrical in nature due to the dissimilar disk rotation velocities. An increase in rotational Reynolds number is observed to strongly depress radial stream function (fig. 4) across the gap although the distributions morph from simple parabolic growths (from the lower to the upper disk) to oscillatory profiles with very strong pressure parameter. Forcing of the flow is therefore evidently intensified with greater pressure parameter which disturbs the regime. The radial velocities are also significantly stifled in the lower half space with increasing rotational Reynolds numbers (fig. 5) with substantial backflow induced which is amplified in the region near the centre line; however, as we progress towards the upper disk there is an acceleration in radial flow and peak radial velocity is located at the upper disk for maximum pressure parameter value of 5. Fig 6 demonstrates that increasing the rate of radial stretching (of the lower disk) i.e. $A_{1}$, markedly accentuates the radial stream function. There is a corresponding acceleration in the radial flow in fig. 7; however, this pattern is again confined to the lower half space. The stretching of the disk certainly aids in momentum and encourages radial flow at the lower disk. However, this boost in radial momentum at the lower zone of the gap is compensated for with a depletion in radial momentum at the upper disk region. The re-distribution is however not identical and the acceleration in the lower disk area is significantly stronger than the deceleration in the upper disk region - this is representative of asymmetric flow structures in differential disk rotation systems. The backflow in the upper half space of the gap is distinctly present and of course strongest at the upper disk surface with highest $R e$ value of 1.6 for which the rotational inertial force is greatest compared with the viscous hydrodynamic force in the regime. Significant manipulation in the structure of the gap flow is therefore achieved by alteration in the rotational velocity of the lower disk and adjustment of the pressure effect in the regime.

\section{Tangential flow characteristics}

Figs 8 and 9 depict the tangential velocity distributions with variation in rotational Reynolds numbers $(R e)$ and relative rotation rate $(\tau)$. Notable deceleration in the tangential flow arises throughout the gap i.e. across the entire depth from the upper to the lower disk. The dual rotating disk system acts much like a fan system drawing fluid axially inward from the surrounding medium towards the lower disk surface. Since no transpiration occurs at this disk surface i.e. it is solid (suction= injection $=0$ ), therefore the incoming fluid is re-directed turned and discharged 
in the radial direction along the lower disk. This accelerates the radial flow and since conservation of momentum cannot be violated, there is a corresponding reduction in tangential momentum, as confirmed in the tangential flow depletion in fig. 8 with greater lower disk-based rotational Reynolds number values. However, no backflow is induced in the tangential field as testified to by the consistently positive values of $g(\eta)$. Fig $\mathbf{9}$ shows that with an increase in relative rotation rate $(\tau)$ there is a significant elevation in tangential velocity magnitudes which again are consistently positive in nature throughout the gap. For the case $\tau=0$, the upper disk is stationary $\left(\Omega_{2} \rightarrow 0\right)$ and only the lower disk rotates - therefore tangential velocity is a minimum for this scenario since there is an absence in the contribution (Coriolis force) from the upper disk. For $\tau>0$, both disks co-rotate and there is a progressive acceleration in tangential flow. The rotational velocity of the lower disk relative to that of the upper disk steadily increases as $\tau$ increases from 0.2 through 0.4 to 0.6 ; however, the case of equal disk rotational velocities $(\tau=1)$ is not considered as it is not relevant to bioreactor designs [1,3,5,7]. Interestingly the tangential flow exhibits a linear decay from the lower disk to the upper disk, which is distinctly different from the parabolic and undulatory profiles characterizing the radial velocity distributions discussed earlier.

\section{Pressure characteristics}

Figs 10, 11 illustrate the response in pressure distribution to a change in rotational Reynolds number $(R e)$ and lower disk radial stretching ratio $\left(A_{1}\right)$. Pressure is weakly depressed in the lower half space (fig. 10) with greater $R e$ values (since radial flow is accelerated there and in viscous or inviscid fluid dynamics there is generally an inverse relationship between pressure and velocity) whereas it is strongly enhanced in the upper half space (where radial flow deceleration arises). Maximum pressure is computed at the upper disk surface for $R e=1.2$ (maximum) whereas the minimum pressure always arises at the lower disk surface. A substantial enhancement in pressure magnitudes accompanies an increase in lower disk radial stretching ratio (fig. 11). The radial stretching generates a pressure ascent from the lower disk to the upper disk (linear profiles) and this can be exploited in rotating disk bioreactor designs to great advantage, as noted by Qiu et al. [9].

\section{Temperature characteristics}

Figs 12, 13 present the evolution in temperature across the gap with a change in values of rotational Reynolds number $(R e)$ and Prandtl number $(P r)$. There is a slight depression in temperature across the gap with a considerable rise in $R e$. The radial flow acceleration implies that momentum diffuses faster and this overcomes thermal diffusion rate in the regime leading to a reduction in transfer of thermal energy in the fluid and a decrease in temperatures. With increasing Prandtl number $\operatorname{Pr}$ there is a more significant depletion in temperatures in particular in the core flow region. Prandtl number signifies the ratio of momentum diffusivity to thermal 
diffusivity. Smaller values of $\operatorname{Pr}$ are equivalent to an increasing thermal conductivity, and therefore heat is able to diffuse away from the heated surfaces (disks) more rapidly than at higher values of Pr. Hence the boundary layer becomes thicker and the rate of heat transfer is reduced at the disk surface. The thermal boundary layer thickness is the distance from the body at which the temperature is $99 \%$ of the temperature found from an inviscid solution. The ratio of the two thicknesses is dictated by the Prandtl number. For Prandtl number of unity, both the hydrodynamic and thermal boundary layers are of the same thickness and the energy and momentum diffusion rates are equal also. However, when Prandtl number exceeds unity, the thermal boundary layer is thinner than the velocity boundary layer (at the disk surfaces) whereas the converse is the case when Prandtl number is less than unity. Generally, higher $\operatorname{Pr}$ fluids will have relatively low thermal conductivities which will suppress thermal conduction heat transfer from the disk surfaces and reduce thermal boundary layer thicknesses at the disks, resulting in lower temperatures throughout the gap region, as demonstrated in Fig. 13. Physically the values of $\operatorname{Pr}=1,4,7,10$ correspond respectively to low molecular weight gases $(1,4)$, water and light lubricants (oils).

\section{Shear stress and heat transfer rate characteristics}

Table-2 presents the perturbation solutions (numerically computed in symbolic software, MATLAB) for lower disk radial $\left[-f^{\prime \prime}(0)\right]$ and tangential $\left[-g^{\prime}(0)\right]$ shear stress and also lower disk surface heat transfer rate (Nusselt number function i.e. $\left.-\theta^{\prime}(0)\right)$ for various values of characterizing parameters. With increasing values of the permeability parameter $(\beta)$ and pressure parameter $(\varepsilon)$. A weak decrease in radial and tangential skin friction at the lower disk is induced with increasing permeability parameter whereas no tangible modification in heat transfer rate is generated. A strong enhancement in radial skin friction at the lower disk is generated with an increase in pressure parameter. With decreasing Prandtl number there is a strong increase in radial skin friction at the lower disk and a weak elevation in tangential skin friction. However there is a marked increase in heat transfer rate at the lower disk surface with a reduction in Prandtl number. The radial and tangential skin friction components are both elevated with increasing values of stretching parameter $\left(A_{l}\right)$ and decrease with greater relative rotational parameter $(\tau)$. Additionally, it is evident that increasing non-Fourier (thermal relaxation) parameter $(\lambda)$ there is a decrease in the rate of heat transfer i.e. the Nusselt number function decreases. This is attributable to the fact that as thermal relaxation time increases the viscous fluid takes longer to transfer thermal energy to the walls (disk surfaces). Hence the non-Fourier model demonstrates that when thermal relaxation effects are neglected (as in the Fourier model) heat transfer -rates are over-predicted and temperatures within the viscous fluid are also underpredicted. 


\section{CONCLUSIONS}

Perturbation solutions have been presented for non-dimensional, coupled, nonlinear ordinary differential boundary value problem describing the steady-state Newtonian thermal convection flow in the homogenous, high permability, porous medium-filled gap between two stretchable spinning disks rotating at different velocities with the Cattaneo-Christov non-Fourier heat flux model. The Darcy porous media drag has been employed which is valid for low Reynolds number regimes. Verification of the perturbation method solutions has been conducted against the earlier (although phsyically limited) homotopy analysis solutions of Hayat et al. [64]. A parametric study of the influence of emerging hydrodynamic, thermophysical and geometric parameters on flow and heat transfer characteristics has been conducted. The computations, which are relevant to the transport phenomena in rotating disk bioreactors, have shown that:

- With increasing rotational Reynolds number (corresponding to greater intensity of rotation of the lower disk), the radial flow is strongly accelerated in the disk gap lower half space whereas it is decelerated in the upper half space.

- With increasing rotational Reynolds number a strong retardation in tangential flow is induced across the entire gap between the disks.

- With greater pressure parameter there is a strong radial flow deceleration in the lower half space of the gap and a significant acceleration in the upper half space which is maximized at the upper disk surface.

- Greater radial stretching of the lower disk induces strong radial flow acceleration (and also an elevation in hydrodynamic pressure) in the lower half space with significant deceleration in the upper half space.

- With increasing relative rotation rate a gradually weaker linear decay is computed in tangential velocity from the lower disk to the upper disk.

- Temperature is weakly reduced with greater rotational Reynolds number and more significantly decreased with increasing Prandtl number.

- Heat transfer rate (Nusselt number function) at the lower disk is reduced with greater non-Fourier (thermal relaxation parameter) effect owing to the delay in thermal diffusion in the fluid.

- The present analysis is of relevance in rotating disk bio-reactors, thermal rheometry and mixing processes in chemical engineering systems. 
- A weak decrease in radial and tangential skin friction at the lower disk is induced with increasing permeability parameter whereas there is no substantial alteration in heat transfer rate.

- A strong enhancement in radial skin friction at the lower disk is generated with an increase in pressure parameter.

- With decreasing Prandtl number there is a strong increase in radial skin friction at the lower disk and a weak elevation in tangential skin friction. However there is a marked increase in heat transfer rate at the lower disk surface with a reduction in Prandtl number.

- The radial and tangential skin friction components are noticeably enhanced with increasing values of lower disk stretching parameter and decrease with greater relative rotational parameter.

The current simulations have considered a no-slip hydrodynamic wall condition for velocity. Future studies will investigate both isotropic and anisotropic hydrodynamic slip [30, 37, 39, 41] and furthermore will also address non-Fourier heat transfer in non-Newtonian fluids e.g. viscoelastic fluids [65], which are of great relevance to rotating disk bioreactor flows in chemical engineering since such systems feature complex fluids. Additionally the current study could be extended to consider variable thickness of the disk [66], chemical reactions [67] and magnetohydrodynamics when the working fluids are electrically-conducting [68].

\section{Declarations}

Availability of Data Material: All data is available with authors only

Competing interests: The author(s) declared no potential conflicts of interest with respect to the research, authorship, and/or publication of this article.

Funding: This research did not receive any specific grant from funding agencies in the public, commercial, or not-for profit sectors.

Authors' contributions: All the authors have contributed equally for the manuscript.

Acknowledgements: This research has not received any specific grants form any sources.

\section{REFERENCES}

[1] Hwang KJ, Lin SJ (2014) Filtration flux-shear stress-cake mass relationships in microalgae rotating-disk dynamic microfiltration. Chem Eng J, 244:429-437.

[2] Zueco J, Anwar Bég O (2010) Network numerical analysis of hydromagnetic squeeze film flow dynamics between two parallel rotating disks with induced magnetic field effects. Tribol Int, 43: 532-543. 
[3] Chen Y, Zhao X, Guan W, Cao D, Guo T, Zhang X, Wang Y (2017) Photoelectrocatalytic oxidation of metalEDTA and recovery of metals by electrodeposition with a rotating cathode. Chem Eng J, 324:74-82.

[4] Rashidi MM, Kavyani N, Anwar Bég O, Gorla RSR (2013) Transient magneto-hydrodynamic film flow, heat transfer and entropy generation from a spinning disk system: DTM-Padé semi-numerical simulation. Int J Energy \& Tech, 5(18):1-14.

[5] Li K, Zhang H, He Y, Tang T, Ying D, Wang Y, Sun T, Jia J (2015) Novel wedge structured rotating disk photocatalytic reactor for post-treatment of actual textile wastewater. Chem Eng J, 268:10-20.

[6] Dickerson JP, Flesher LM, Hester RD (1994) A high Reynolds number rotating disk rheometer. J Appl Polymer Sci, 53:151-159.

[7] Zanfir M, Sun X, Gavriilidis A (2007) Investigation of a rotating disc reactor for acetone stripping and asymmetric transfer hydrogenation: Modelling and experiments. Chem Eng Sci, 62: 741-755.

[8] Anwar Bég O, Zueco J, López-Ochoa LM (2011) Network numerical analysis of optically-thick hydromagnetic slip flow from a porous spinning disk with radiation flux, variable thermophysical properties and surface injection effects. Chem Eng Commun, 198(3):360-384.

[9] Qiu Z, Petera J, Weatherley LR (2012) Biodiesel synthesis in an intensified spinning disk reactor. Chem Eng J, 210:597-609.

[10] Mantripragada VT, Sarkar S (2017) Prediction of drop size from liquid film thickness during rotary disc atomization process. Chem Eng Sci, 158: 227-233.

[11]Lance GN, Rogers MH (1962) The axially symmetric flow of a viscous fluid between two infinite rotating disks. Proc Roy Soc A:Mathematical, Physical and Eng Sci, 266:109-130.

[12] Mellor GL, Chapple PJ, Stokes VK (1968) On the flow between a rotating and a stationary disk. J Fluid Mech 31:95-112.

[13] Srinivasan S, Karra S (2015) Flow of stress power-law fluids between parallel rotating discs with distinct axes. Int J Non-Linear Mech, 74:73-83.

[14] Ersoy HV (1999) MHD Flow of an Oldroyd-B fluid between eccentric rotating disks. Int J Eng Sci, 37:19731984.

[15] Sha Y, Dillenburger T, Fritz J, Klemm T, Ripperger S (2016) Investigation of the flow character in the gap between a rotating disk and a static opposite disk. Chemie Ingenieur Technik, 88: 1-10.

[16] Anwar Bég O, Tripathi D, Sochi T, Gupta PK (2015) Adomian decomposition method (ADM) simulation of magneto-bio-tribological squeeze film with magnetic induction effects. J Mech Med Biol, 15: 1550072.11550072.23

[17] Sparrow EM, Gregg JL (1960) Mass transfer, flow and heat transfer about a rotating disk. ASME J Heat Transfer, 82(4): 294-302.

[18] Dorfman LA (1963) Hydrodynamic Resistance and the Heat Loss of Rotating Solids, Oliver \& Boyd, New York.

[19] Arora RC, Stokes VK (1972) On the heat transfer between two rotating disks. Int J Heat and Mass Transfer, 15: 2119-2132.

[20] Hudson JL (1968) Non-isothermal flow between rotating disks. Chem Eng Sci, 23:1007-1020.

[21] Ming C, Zheng L, Zhang X (2011) Steady flow and heat transfer of the power-law fluid over a rotating disk. Int Commun Heat and Mass Transfer, 38:280-284. 
[22] Yen SC, Wang JS (1992) Mass transfer and fluid flow due to a rotating disk with external forced convection. Chem Eng Sci, 47:2001-2008.

[23]Rahman MM, Postelnicu A (2010) Effects of thermophoresis on the forced convective laminar flow of a viscous incompressible fluid over a rotating disk. Mech Res Commun 37:598-603.

[24] Sahoo B, Van Gorder RA, Andersson HI (2012) Steady revolving flow and heat transfer of a non-Newtonian Reiner-Rivlin fluid. Int Commun Heat and Mass Transfer, 39:336-342.

[25] Basu S, Cetegen BM (2005) Analysis of hydrodynamics and heat transfer in a thin liquid film flowing over a rotating disk by the integral method. ASME J Heat Transfer, 28(3):217-225.

[26] Helcig C, Stefan aus der Wiesche, Shevchuk IV (2017) Prandtl number effect on the laminar convective heat transfer from a rotating disk. ASME J Heat Transfer, 139(10):101702-1-101702-10.

[27] Jiji LM, Ganatos P (2010) Microscale flow and heat transfer between rotating disks. Int J Heat and Fluid Flow, 31: 702-710.

[28] Weinstein SJ, Gros A (2005) Viscous liquid sheets and operability bounds in extrusion coating. Chem Eng Sci, 60:5499-5512.

[29] Termonia Y (1997) Fundamental study of the deformation behavior of nonwoven heat-bonded sheets. Chem Eng Sci, 52:3003-3009.

[30] Khan M, Hashim, Hafeez A (2017) A review on slip-flow and heat transfer performance of nanofluids from a permeable shrinking surface with thermal radiation: Dual solutions. Chem Eng Sci, 173:1-11.

[31] Ali L, Islam S, Gul T, Alshomrani AS, Khan I, Khan A (2017) Magnetohydrodynamics thin film fluid flow under the effect of thermophoresis and variable fluid properties. AIChE J, 63:200-210.

[32] Anwar Bég O, Bakier AY, Prasad VR (2009) Numerical study of free convection magnetohydrodynamic heat and mass transfer from a stretching surface to a saturated porous medium with Soret and Dufour effects. Comput Mat Sci, 46(1):57-65.

[33] Abel MS, Tawade J, Mahantesh MN (2009) Effect of non-uniform heat source on MHD heat transfer in a liquid film over an unsteady stretching sheet. Int J Non-Linear Mech, 44(9):990-998.

[34] Ahmad N, Siddiqui ZU, Mishra MK (2010) Boundary layer flow and heat transfer past a stretching plate with variable thermal conductivity. Int J Non-Linear Mech, 45:306-309.

[35] Gupta D, Kumar L, Anwar Bég O, Singh B (2014) Finite element analysis of transient heat and mass transfer in microstructural boundary layer flow from porous stretching sheet. Comput Therm Sci, 6(2):155-169.

[36] Yam KS, Harris SD, Ingham DB, Pop I (2009) Boundary-layer flow of Reiner-Philippoff fluids past a stretching wedge. Int J Non-Linear Mech, 44(10):1056-1062.

[37] Uddin MJ, Anwar Bég O, Ismail AIM (2015) Radiative-convective nanofluid flow past a stretching/shrinking sheet with slip effects. AIAA J Thermophysics Heat Transfer, 29(3):513-523.

[38] Sajid M, Ali N, Javed T, Abbas Z (2010) Stretching a curved surface in a viscous fluid. Chin Phys Letters, 27:024703-1-024703-4.

[39] Latiff NA, Uddin MJ, Anwar Bég O, Ismail AIM (2016) Unsteady forced bioconvection slip flow of a micropolar nanofluid from a stretching/ shrinking sheet. Proc IMechE- Part N: J Nanoengineering and Nanosystems, 230(4):177-187.

[40] Hayat T, Saeed Y, Asad S, Alsaedi A (2016) Convective heat and mass transfer in flow by an inclined stretching cylinder. J Molecular Liquids, 220:573-580. 
[41] Anwar Bég O, Basir MFM, Uddin MJ, Ismail AIM (2017) Numerical study of slip effects on asymmetric bioconvective nanofluid flow in a porous microchannel with an expanding/contracting upper wall using Buongiorno's model. J Mech Med Biol, 17(5):1750059-1-1750059-28.

[42] Ali ME (1995) On thermal boundary layer on a power-law stretched surface with suction or injection. Int J Heat and Fluid Flow, 16:280-290.

[43] Basir MFM, Uddin MJ, Ismail AIM, Anwar Bég O (2016) Unsteady bio-nanofluid slip flow over a stretching cylinder with bioconvection Schmidt and Péclet number effects. AIP Advances, 6:055316-1 - 055316-15.

[44] Anwar Bég O, Rashidi MM, Mehr NF (2013) Second law analysis of hydromagnetic flow from a stretching rotating disk: DTM-Padé simulation of novel nuclear MHD propulsion systems. Frontiers of Aerospace Eng, 2(1):29-38.

[45] Hashmi MS, Khan N, Mahmood T, Shehzad SA (2017) Effect of magnetic field on mixed convection flow of Oldroyd-B nanofluid induced by two infinite isothermal stretching disks. Int J Thermal Sci, 111:463-474.

[46]Fang T, Zhang J (2008) Flow between two stretchable disks-An exact solution of the Navier-Stokes equations. Int Commun Heat and Mass Transfer, 35:892-895.

[47] Yin C, Zheng L, Zhang C, Zhang X (2017) Flow and heat transfer of nanofluids over a rotating disk with uniform stretching rate in the radial direction. Propulsion and Power Research, 6(1):25-30.

[48]Ellahi R, Tariq MH, Hassan M, Vafai K (2017) On boundary layer nano-ferroliquid flow under the influence of low oscillating stretchable rotating disk. J Molecular Liquids, 229:339-345.

[49] Hayat T, Javed M, Imtiaz M, Alsaedi A (2017) Convective flow of Jeffrey nanofluid due to two stretchable rotating disks. J Molecular Liquids, 240:291-302.

[50] Cattaneo C (1948) Sulla conduzione del calore, Atti Semin Mat Fis Bull Univ Modena, 3: 83-101.

[51]Frankel JI, Vick B, Ozisik MN (1985) Flux formulation of hyperbolic heat conduction. J Appl Phys, 58(9): 3340-3345.

[52] Mishra SC, Stephen A, Kim MY (2010) Analysis of non-Fourier conduction-radiation heat transfer in a cylindrical enclosure. Numerical Heat Transfer, Part A: Applic, 58(12):943-962.

[53] Roetzel W, Putra N, Das SK (2003) Experiment and analysis for non-Fourier conduction in materials with non-homogeneous inner structure. Int J Thermal Sci, 42:541-552.

[54]Dai SC, Tanner RI (2006) Anisotropic thermal conductivity in sheared polypropylene. Rheologica Acta, 45: 228-238.

[55] Auriault JL (2017) The paradox of Fourier heat equation: A theoretical refutation. Int J Eng Sci, 118:82-88.

[56] Han S, Peddieson J (2018) Non-Fourier heat conduction/convection in moving medium. Int J Thermal Sci, 130:128-139.

[57]Elsayed AF, Anwar Bég O (2014) New computational approaches for biophysical heat transfer in tissue under ultrasonic waves: Variational iteration and Chebyshev spectral simulations. J Mech Med Biol, 14(3): 1450043.1-1450043.17.

[58] Liu KC, Chen HT, Cheng PJ (2016) Inverse investigation of non-Fourier heat conduction in tissue. J Thermal Biol, 62B:123-128.

[59]Herwig H, Beckert K (1999) Fourier versus non-Fourier heat conduction in materials with a nonhomogeneous inner structure. ASME J Heat Transfer, 122(2):363-365. 
[60] Singh S, Kumar D, Rai KN (2018) Analytical solution of Fourier and non-Fourier heat transfer in longitudinal fin with internal heat generation and periodic boundary condition. Int J Thermal Sci, 125:166-175.

[61] Ai X, Li BQ (2003) A Discontinuous finite element method for non-Fourier heat conduction problems. ASME 2003 International Mechanical Engineering Congress and Exposition, Heat Transfer, Volume 1, Washington, DC, USA, November 15-21.

[62] Akbar NS, Anwar Bég O, Khan ZH (2017) Magneto-nanofluid flow with heat transfer past a stretching surface with a new heat flux model using numerical approach. Int J Numerical Methods for Heat and Fluid Flow, 27(6):1-17.

[63] Shahid A, Bhatti MM, Anwar Bég O, Kadir A (2017) Numerical study of radiative Maxwell viscoelastic magnetized flow from a stretching permeable sheet with the Cattaneo-Christov heat flux model, Neural Comput \& Applic. https://doi.org/10.1007/s00521-017-2933-8.

[64] Hayat T, Qayyum S, Imtiaz M, Alsaedi A (2017) Flow between two stretchable rotating disks with CattaneoChristov heat flux model. Results in Phys, 7:126-133.

[65] Mehmood R, Rana S, Anwar Bég O, Kadir A (2018) Numerical study of chemical reaction effects in magnetohydrodynamic Oldroyd B oblique stagnation flow with a non-Fourier heat flux model. Int J Eng Sci, (Communicated).

[66] T. Hayat, M.I. Khan, M. Farooq, A. Alsaedi, M. Waqas and T. Yasmeen, Impact of Cattaneo-Christov heat flux model in flow of variable thermal conductivity fluid over a variable thicked surface, International Journal of Heat and Mass Transfer, 99, 702-710 (2016).

[67] T. Hayat, M.I. Khan, M. Farooq, T. Yasmeen, A. Alsaedi, Stagnation point flow with Cattaneo-Christov heat flux and homogeneous-heterogeneous reactions, Journal of Molecular Liquids, 220, 49-55 (2016).

[68] T. Hayat, S. Qayyum, M.I. Khan, A. Alsaedi Entropy generation in magnetohydrodynamic radiative flow due to rotating disk in presence of viscous dissipation and Joule heating, Physics of Fluids 30, 017101 (2018); https://doi.org/10.1063/1.5009611 


\section{TABLES}

Table-1: Comparison table for various values of pertinent parameters with HAM solutions of Hayat et al. [65]

\begin{tabular}{|c|c|c|c|c|c|c|c|c|c|c|}
\hline$\beta$ & $R e$ & $\varepsilon$ & $P r$ & $A_{1}$ & $\tau$ & $\lambda$ & $\begin{array}{c}-g^{\prime}(0) \\
\text { HAM [65] }\end{array}$ & $\begin{array}{c}\text { Perturbation } \\
\text { Solution }\end{array}$ & $\begin{array}{c}-\theta^{\prime}(0) \\
\text { HAM [65] }\end{array}$ & $\begin{array}{c}\text { Perturbation } \\
\text { Solution }\end{array}$ \\
\hline 0.9 & 0.01 & 5 & 0.7 & 0.2 & 0.8 & 0.2 & 0.204878519 & 0.204473994 & 0.99993028 & 0.9998387 \\
\hline
\end{tabular}

Table-2: Radial shear stress, tangential shear stress and wall heat transfer rate variation with all key parameters

\begin{tabular}{|c|c|c|c|c|c|c|c|c|c|}
\hline$\beta$ & $R e$ & $\varepsilon$ & $P r$ & $A_{1}$ & $\tau$ & $\lambda$ & $-f^{\prime \prime}(0)$ & $-g^{\prime}(0)$ & $-\theta^{\prime}(0)$ \\
\hline 0.9 & 0.01 & 5 & 0.7 & 0.4 & 0.8 & 0.2 & 2.063586 & 0.2044739 & 0.9998387 \\
\hline 1 & & & & & & & 2.06361049 & 0.20395608 & 0.99983868 \\
\hline 1.1 & & & & & & & 2.0636302 & 0.20353227 & 0.99983865 \\
\hline 0.9 & 0.1 & & & & & & 2.03625273 & 0.24473276 & 0.998474622 \\
\hline & 0.2 & & & & & & 2.00670264 & 0.289449591 & 0.997143526 \\
\hline & 0.01 & 4 & & & & & 1.73032617 & 0.204762517 & 0.999920029 \\
\hline & & 3 & & & & & 1.397087102 & 0.205050952 & 0.99999985 \\
\hline & & 5 & 1 & & & & 2.0635864 & 0.20447399 & 0.9997696 \\
\hline & & & 3 & & & & 2.0635864 & 0.2044739 & 0.9993096 \\
\hline & & & 0.7 & 0.4 & & & 2.46413517 & 0.20520703 & 1.0000765 \\
\hline & & & & 0.6 & & & 2.86495 & 0.205939675 & 1.0003068 \\
\hline & & & & 0.2 & 0.9 & & 2.0634301 & 0.104653583 & 0.99983878 \\
\hline & & & & & 1 & & 2.063267 & 0.0048331 & 0.9998388 \\
\hline & & & & & 0.8 & 0.4 & 2.0635864 & 0.2044739 & 0.9998321 \\
\hline & & & & & & 0.6 & 2.0635864 & 0.204473 & 0.9998255 \\
\hline
\end{tabular}




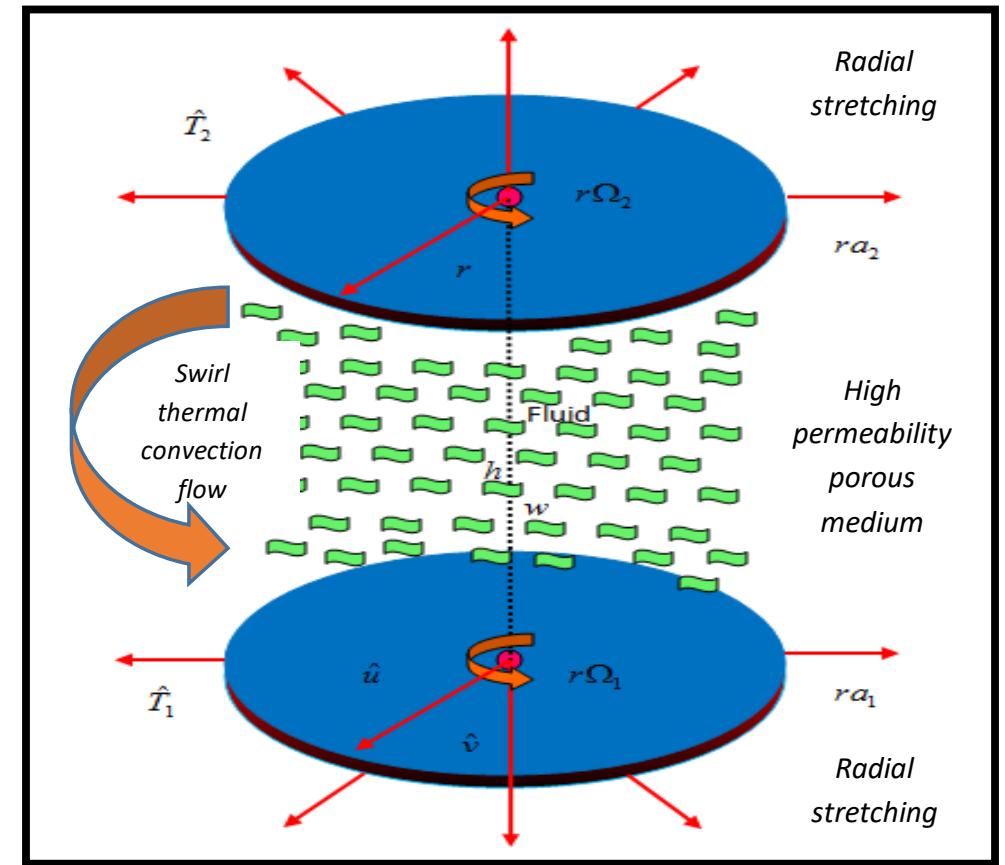

Fig.1 Swirling heat transfer in the porous medium gap between two rotating disks

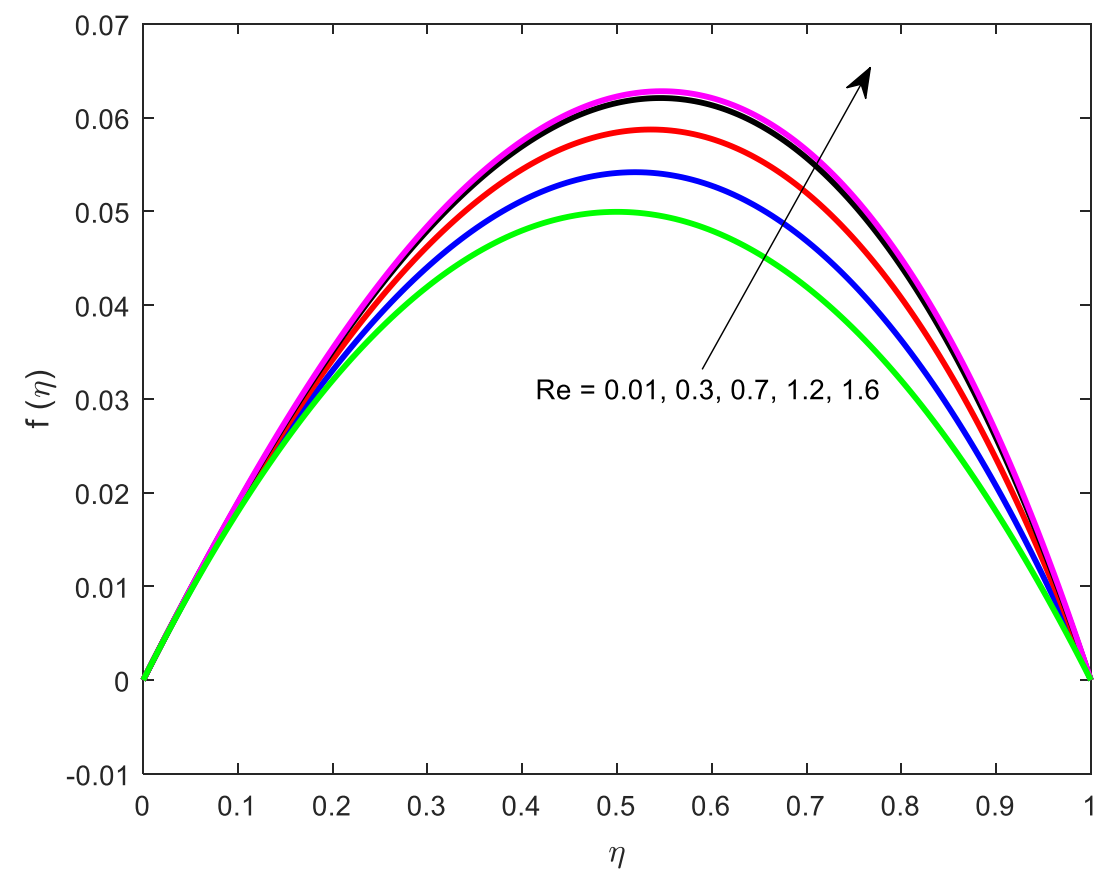

Fig.2 Radial stream function distribution with different rotational Reynolds numbers for $\varepsilon=0.01, \beta=0.9, \operatorname{Pr}=$ 0.7 (air), $A_{l}=0, \tau=0.8, \lambda=0.2$. 


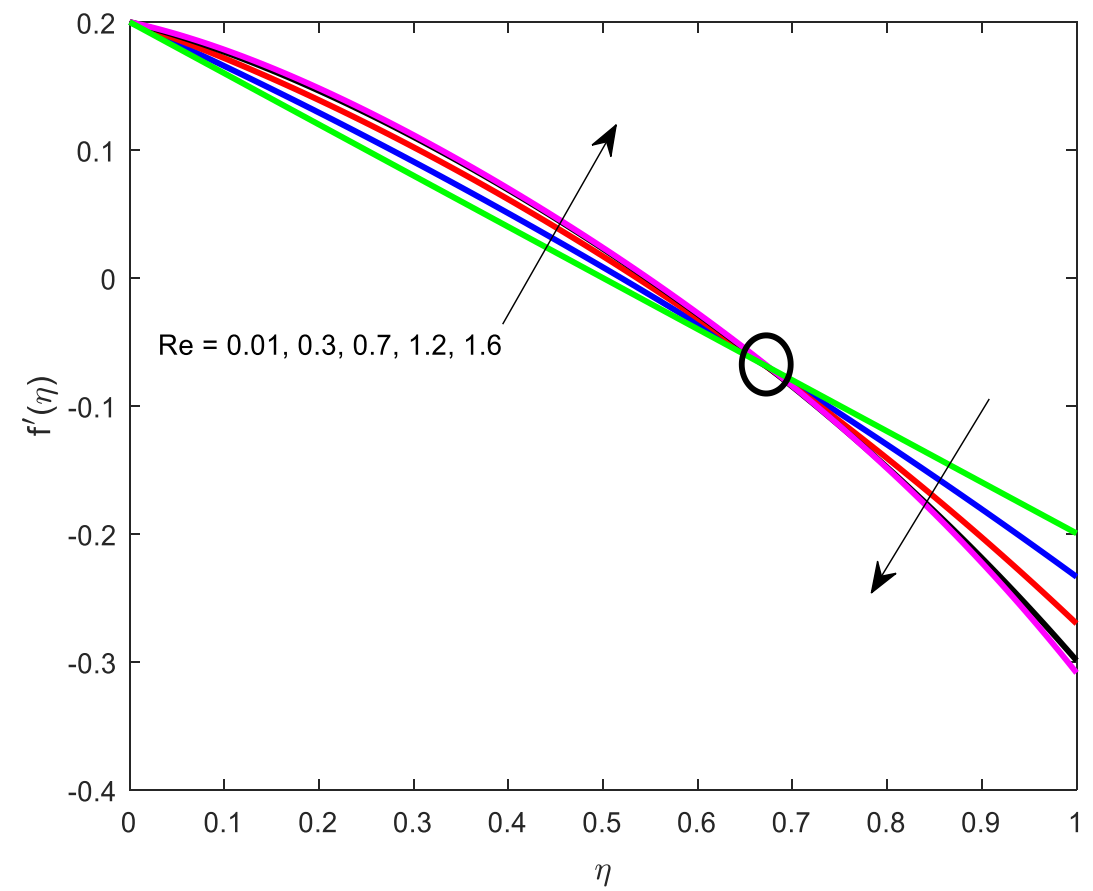

Fig.3 Radial stream function evolution with different rotational Reynolds numbers for $\varepsilon=0.01, \beta=0.9, \operatorname{Pr}=0.7$ (air), $A_{l}=0, \tau=0.8, \lambda=0.2$.

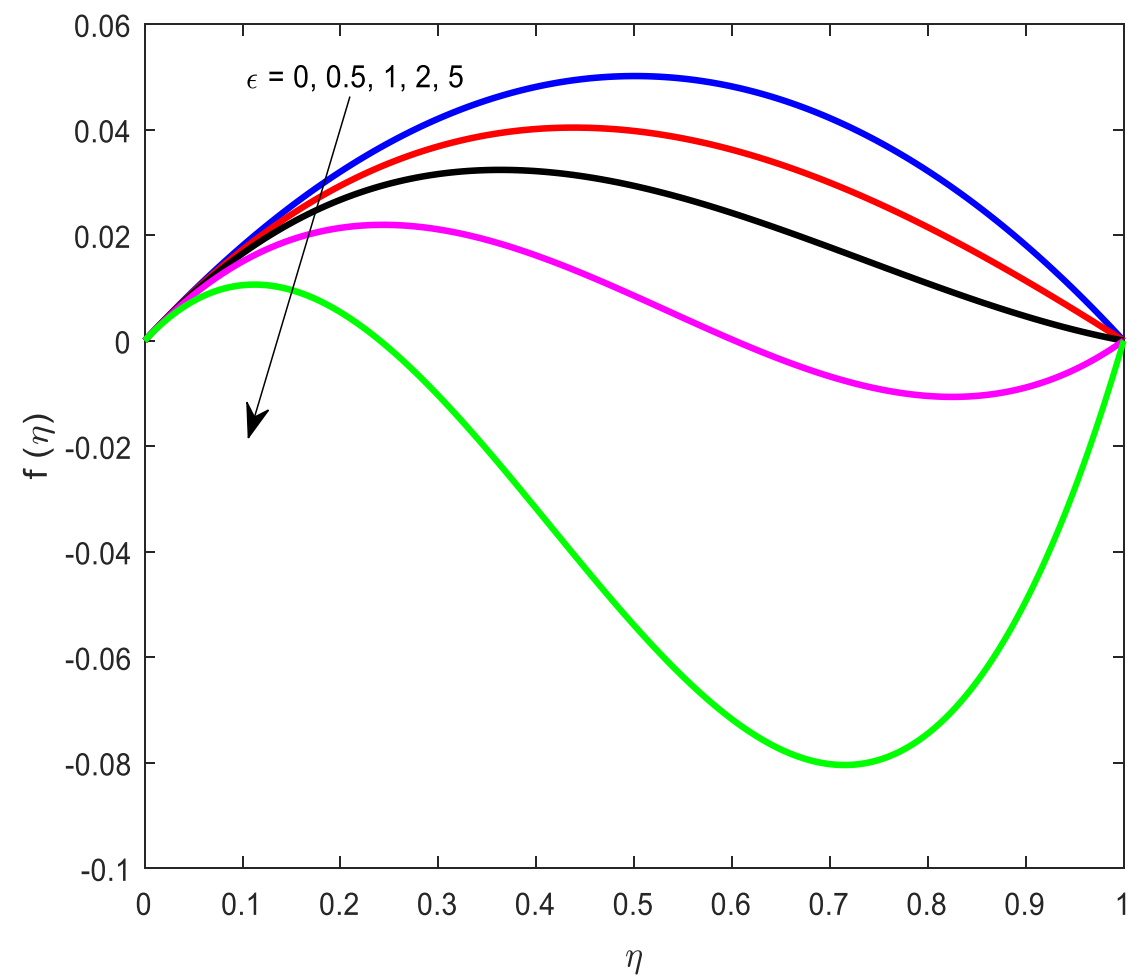

Fig.4 Radial stream function profiles with different pressure parameters for $\operatorname{Re}=0.01, \beta=0.9, \operatorname{Pr}=0.7$ (air), $A_{1}=$ 0.2 (lower disk radial stretching), $\tau=0.8, \lambda=0.2$. 


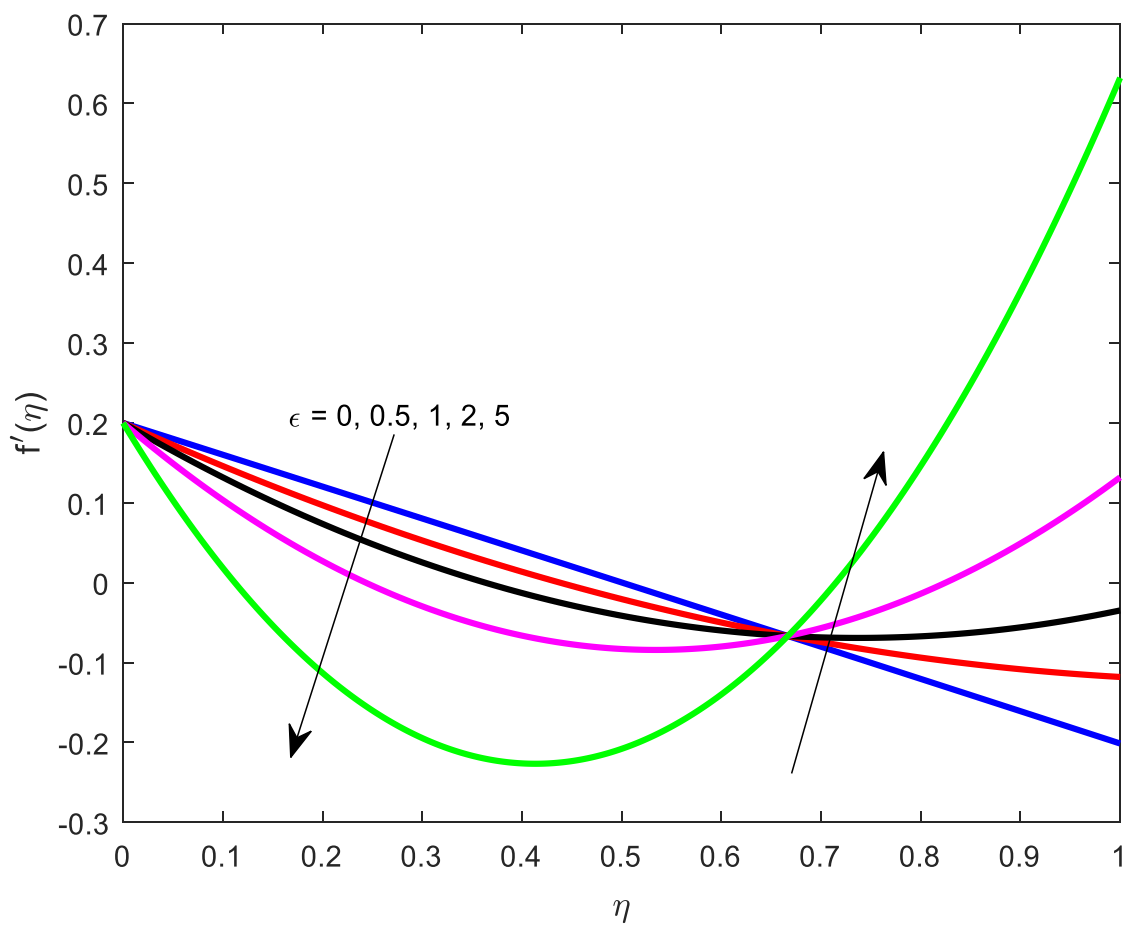

Fig.5 Radial velocity variation with different pressure parameters for $\operatorname{Re}=0.01, \beta=0.9, \operatorname{Pr}=0.7$ (air), $A_{l}=0.2$ (lower disk radial stretching), $\tau=0.8, \lambda=0.2$.

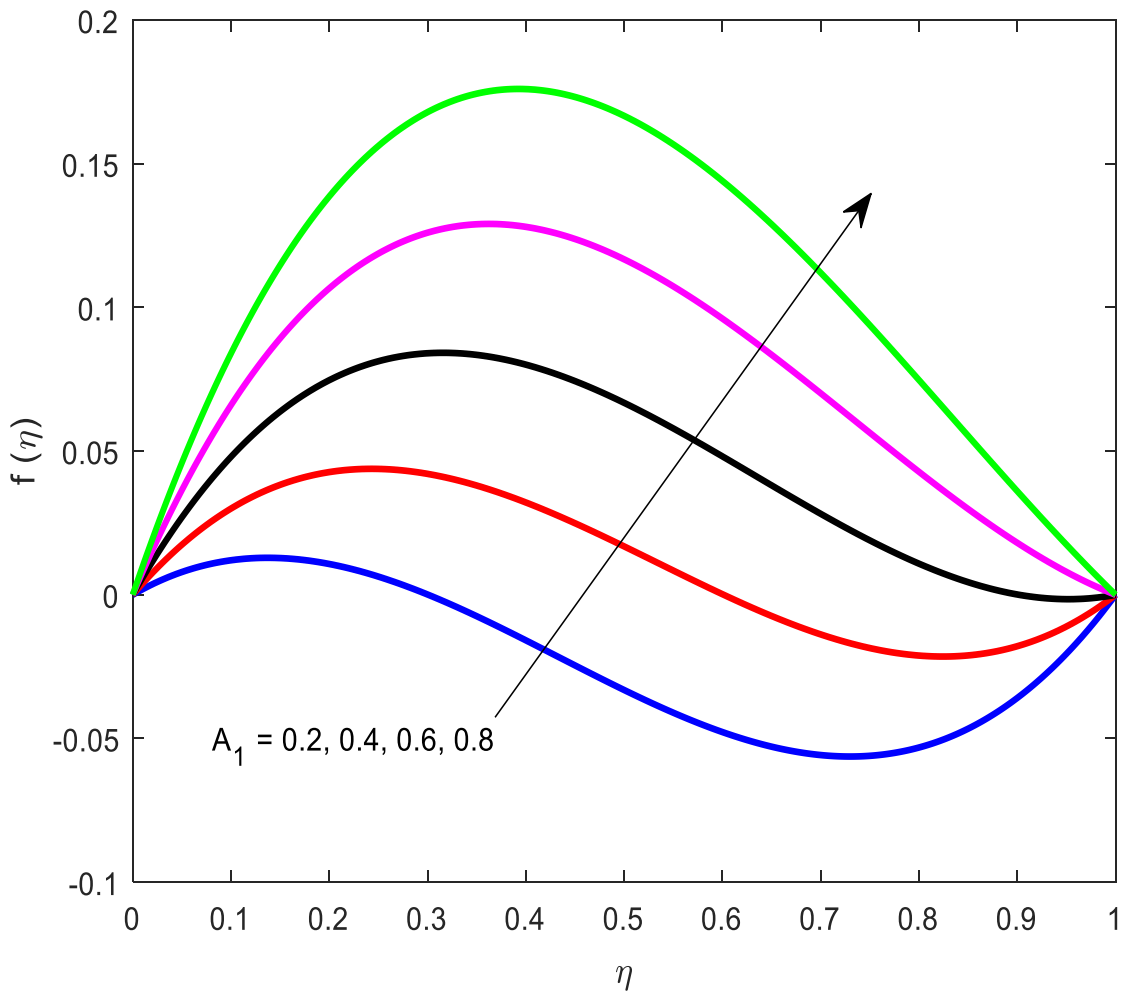

Fig.6 Radial stream function variation with lower disk radial stretching rate for $\operatorname{Re}=0.01, \beta=0.9, \operatorname{Pr}=0.7$ (air), $\varepsilon=4, \tau=0.8, \lambda=0.2$. 


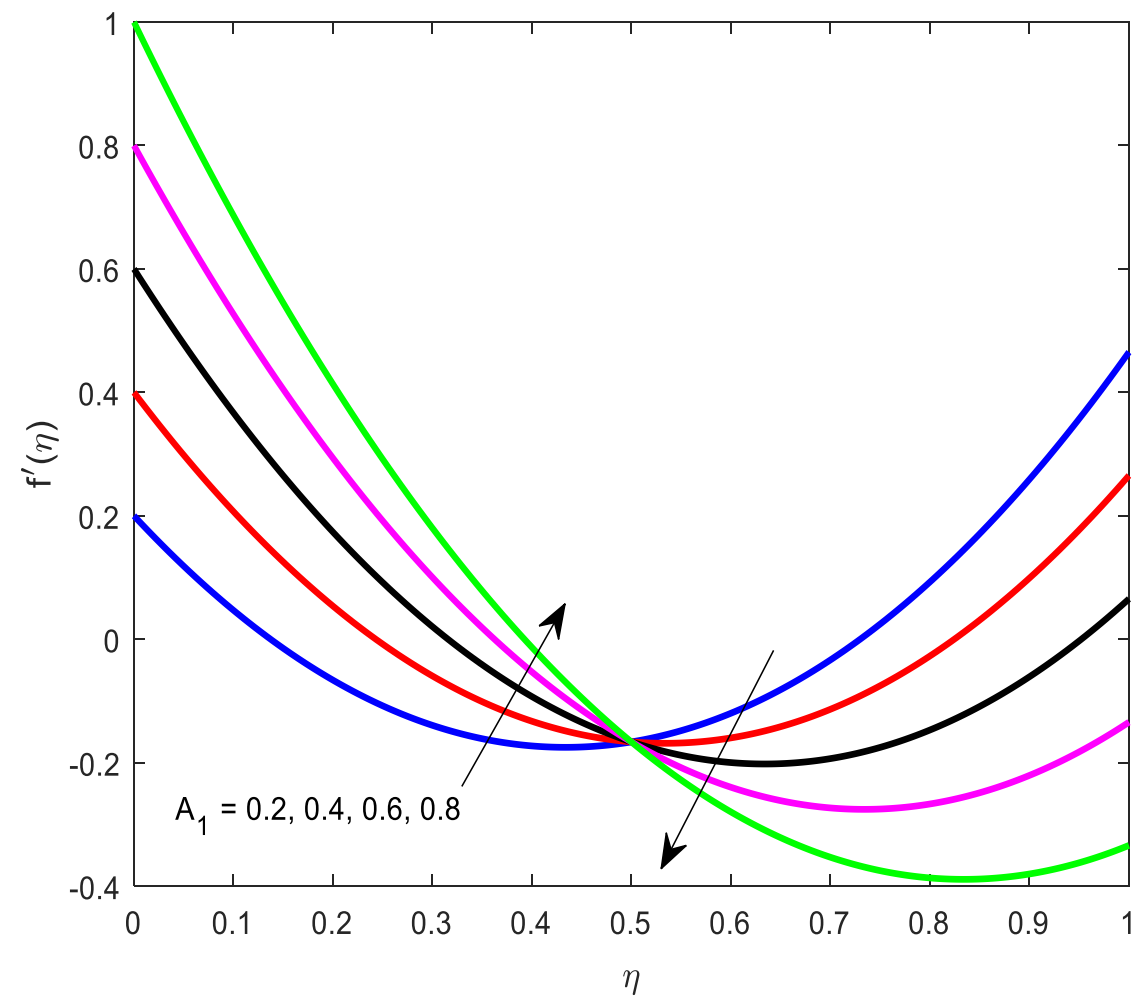

Fig.7 Radial velocity profiles with lower disk radial stretching rate for $\operatorname{Re}=0.01, \beta=0.9, \operatorname{Pr}=0.7$ (air), $\varepsilon=4, \tau=$ $0.8, \lambda=0.2$.

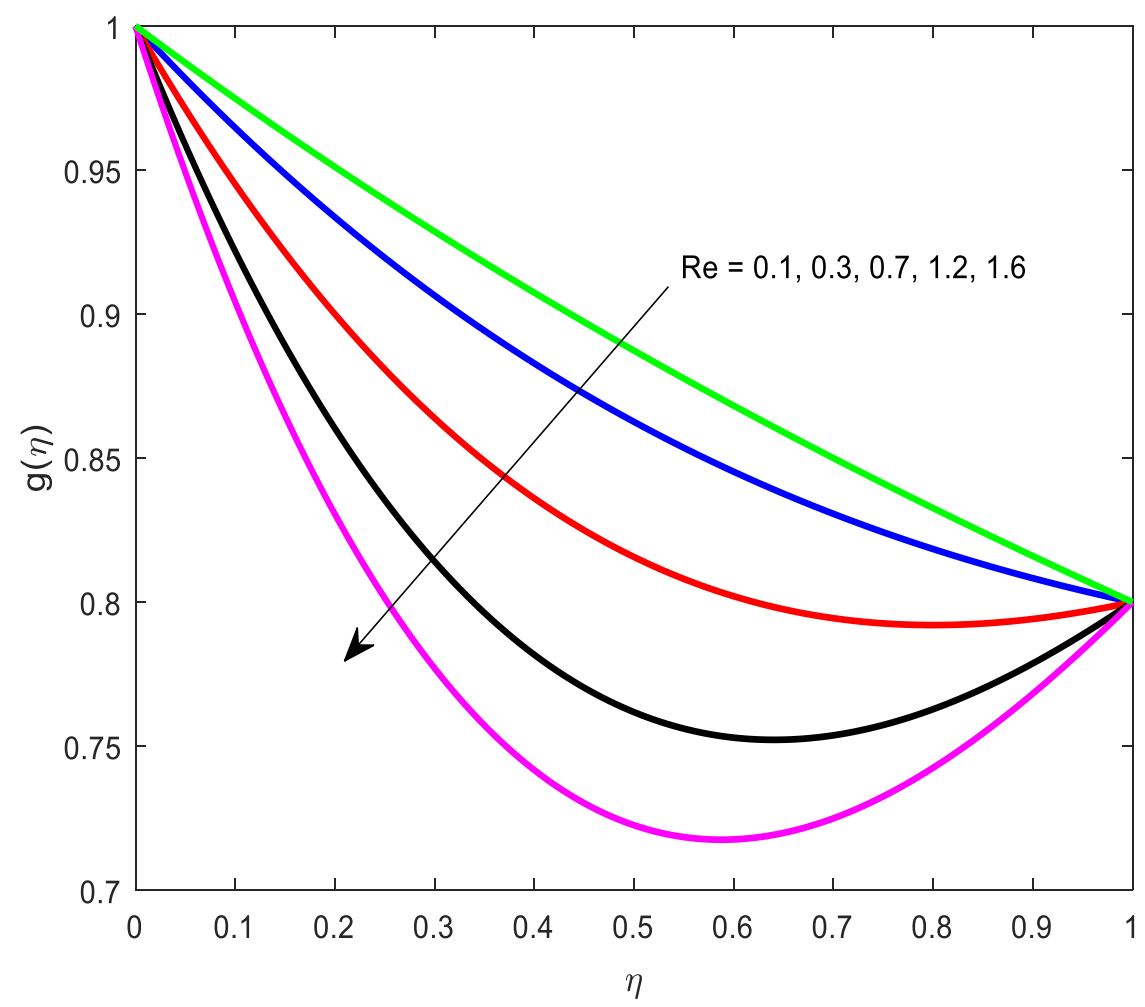

Fig.8 Tangential velocity profiles with different rotational Reynolds numbers for $\varepsilon=0.01, \beta=0.9, \operatorname{Pr}=0.7$ (air), $A_{l}=0.2, \tau=0.8, \lambda=0.2$. 


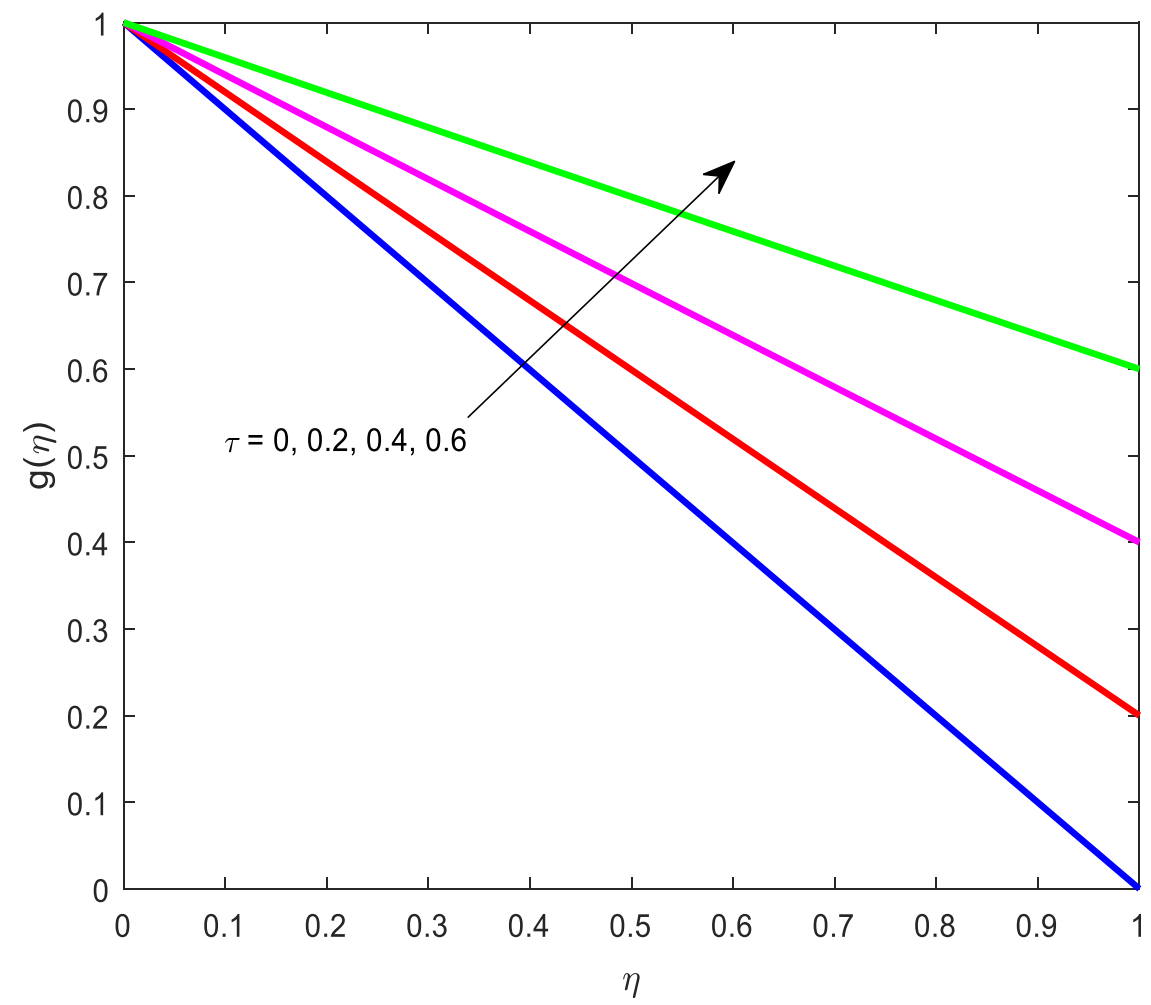

Fig.9 Tangential velocity profiles with different relative rotation rate for $\operatorname{Re}=0.01, \varepsilon=0.01, \beta=0.9, \operatorname{Pr}=0.7$ (air), $A_{l}=0.2, \lambda=0.2$.

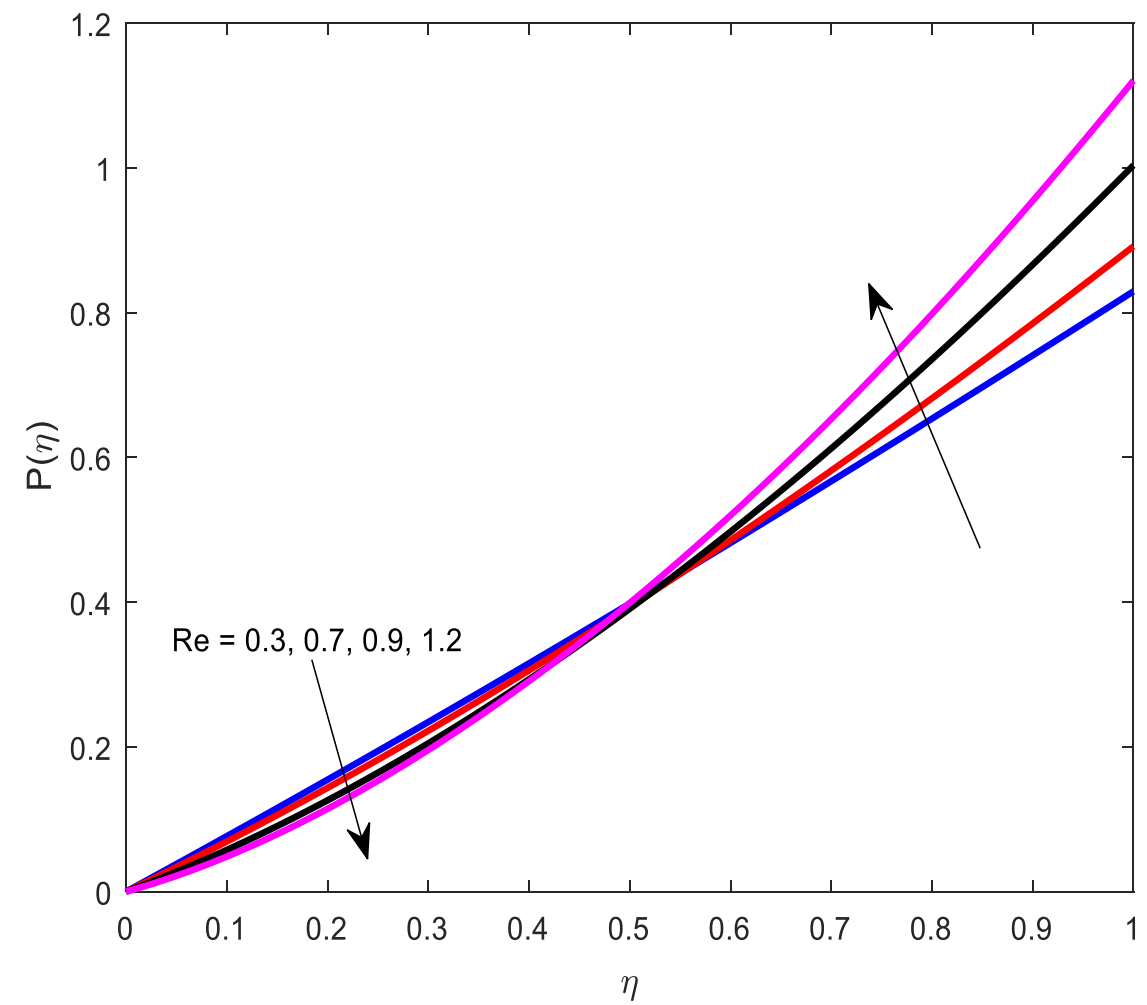

Fig.10 Pressure distribution with different rotational Reynolds numbers for $\varepsilon=0.01, \beta=0.9, \operatorname{Pr}=0.7$ (air), $A_{l}=$ $0.2, \tau=0.8, \lambda=0.2$. 


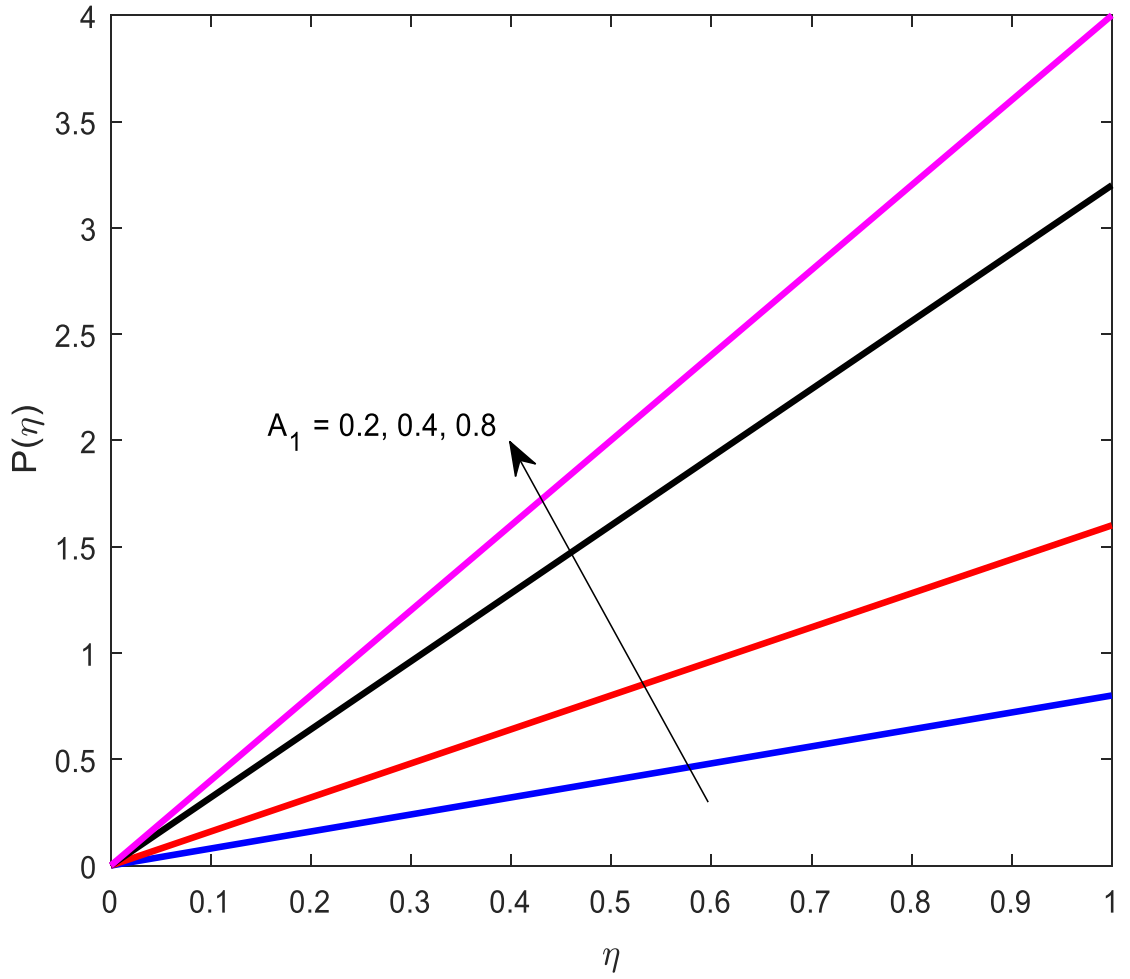

Fig.11 Pressure distribution with different lower disk radial stretching rate for $\varepsilon=0.01, \beta=0.9, \operatorname{Pr}=0.7$ (air), $R e$ $=0.01, \tau=0.8, \lambda=0.2$.

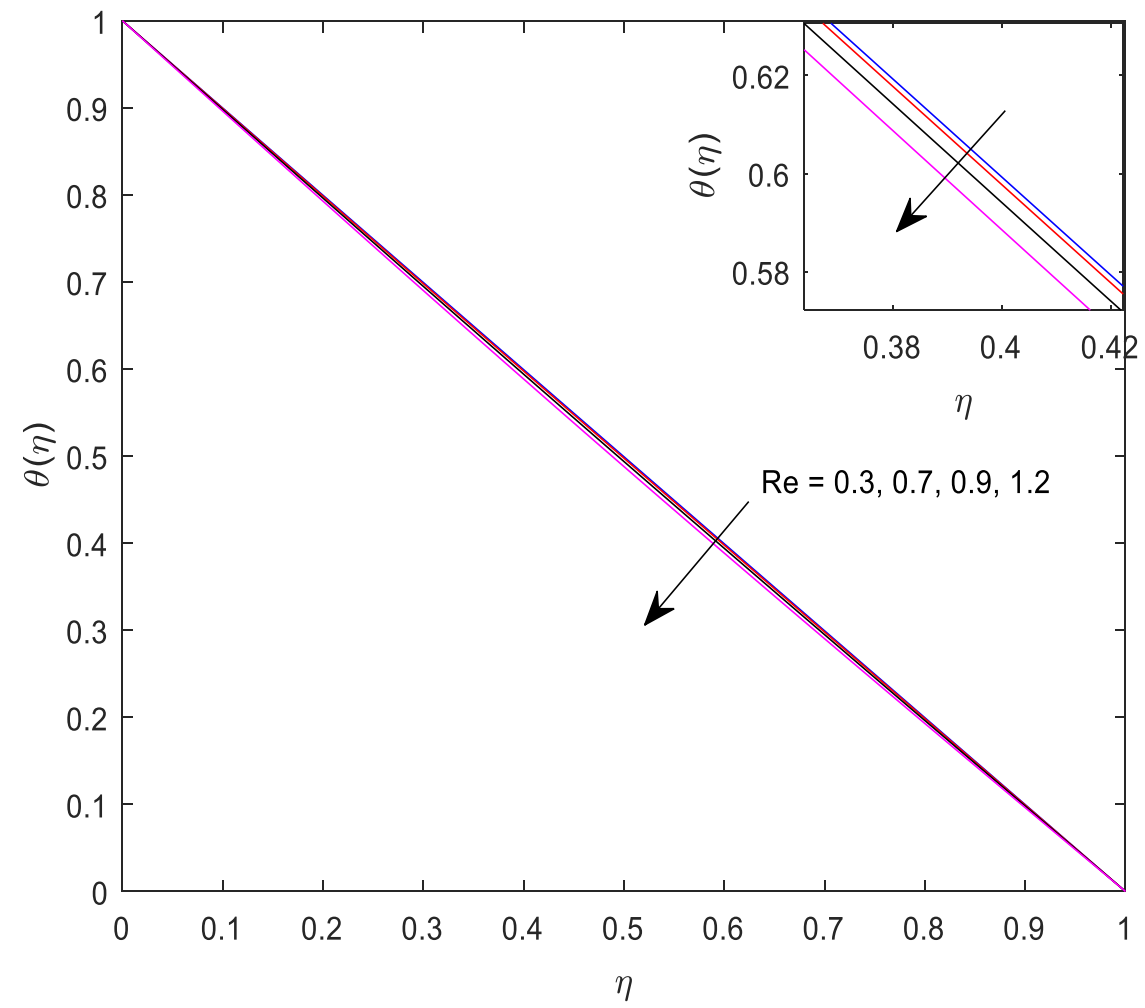

Fig.12 Temperature distributions with different rotational Reynolds numbers for $\varepsilon=0.01, \beta=0.9, \operatorname{Pr}=0.7$ (air), $A_{l}=0.2, \tau=0.8, \lambda=0.2$. 


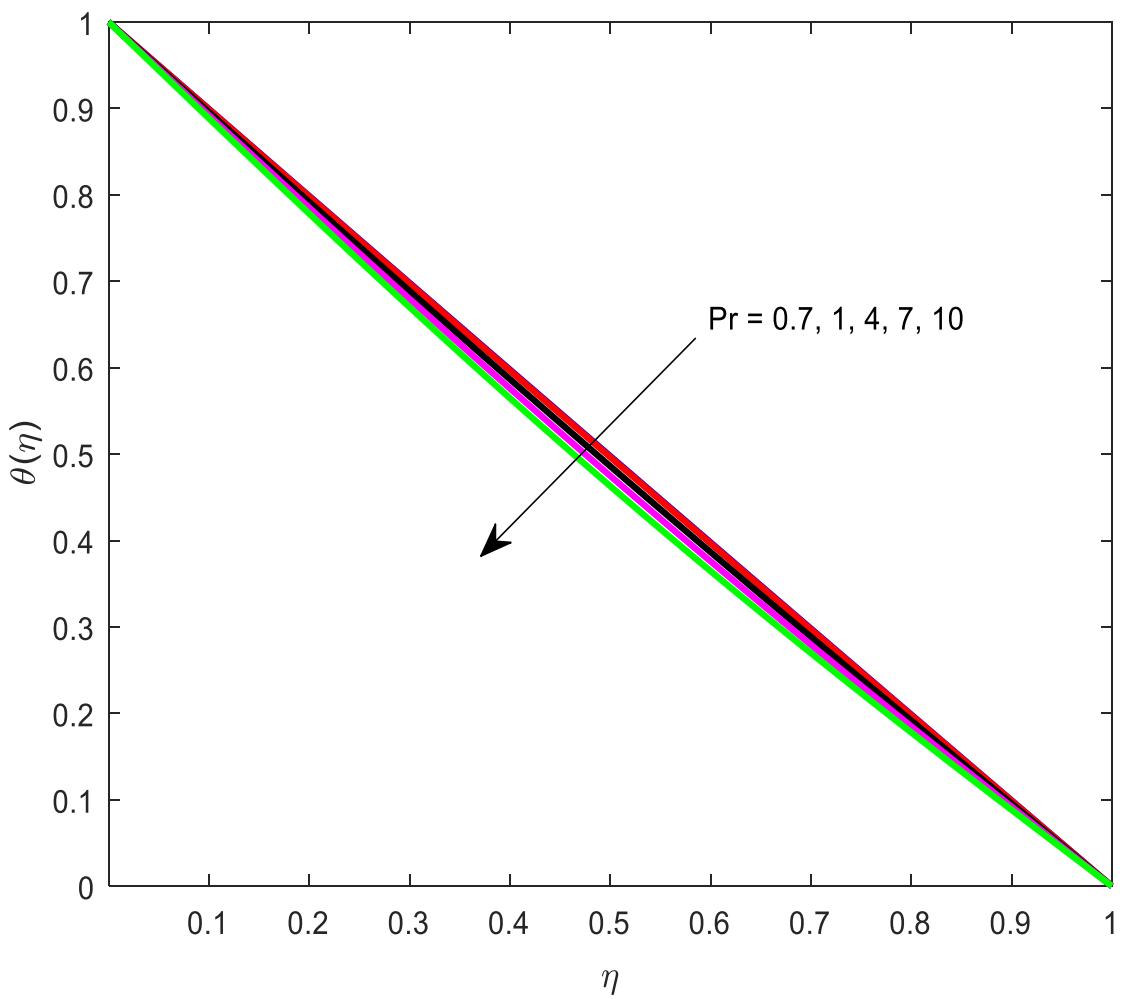

Fig.13 Temperature distributions with different Prandtl numbers for $\varepsilon=0.01, \beta=0.9, R e=0.20 .7$ (air), $A_{l}=$ $0.2, \tau=0.8, \lambda=0.2$. 